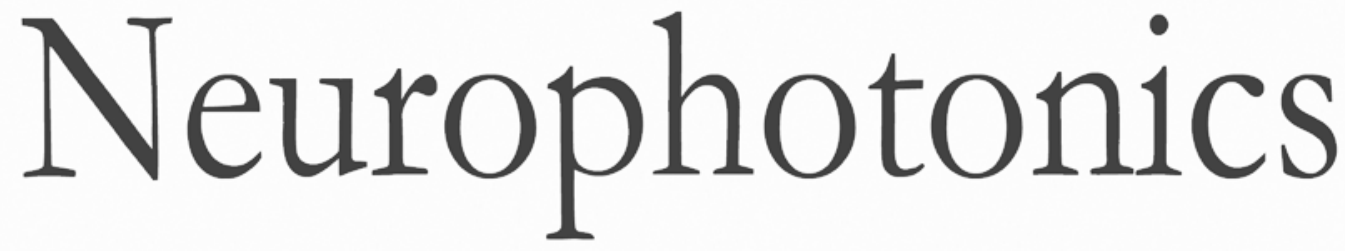

\title{
Density center-based fast clustering of widefield fluorescence imaging of cortical mesoscale functional connectivity and relation to structural connectivity
}

\author{
Miaowen Li \\ Shen Gui \\ Qin Huang \\ Liang Shi \\ Jinling Lu \\ Pengcheng Li
}




\title{
Density center-based fast clustering of widefield fluorescence imaging of cortical mesoscale functional connectivity and relation to structural connectivity
}

\author{
Miaowen Li, ${ }^{a, b}$ Shen Gui, ${ }^{a, b}$ Qin Huang, ${ }^{a, b}$ Liang Shi, ${ }^{a, b}$ Jinling Lu, ${ }^{a, b}$ and Pengcheng $\mathrm{Li}^{\mathrm{a}, \mathrm{b}, \mathrm{c}, \mathrm{c},}$ \\ ${ }^{a}$ Huazhong University of Science and Technology, Wuhan National Laboratory for Optoelectronics, Britton Chance Center for Biomedical \\ Photonics, Wuhan, Hubei, China \\ ${ }^{b}$ Huazhong University of Science and Technology, School of Engineering Sciences, MOE Key Laboratory for Biomedical Photonics, \\ Wuhan, Hubei, China \\ 'HUST-Suzhou Institute for Brainsmatics, Suzhou, China
}

\begin{abstract}
Spontaneous resting-state neural activity or hemodynamics has been used to reveal functional connectivity in the brain. However, most of the commonly used clustering algorithms for functional parcellation are time-consuming, especially for high-resolution imaging data. We propose a density center-based fast clustering (DCBFC) method that can rapidly perform the functional parcellation of isocortex. DCBFC was validated using both simulation data and the spontaneous calcium signals from widefield fluorescence imaging of excitatory neuron-expressing transgenic mice (Vglut2-GCaMP6s). Compared to commonly used clustering methods such as k-means, hierarchical, and spectral, DCBFC showed a higher adjusted Rand index when the signal-to-noise ratio was greater than $-8 \mathrm{~dB}$ for simulated data and higher silhouette coefficient for in vivo mouse data. The resting-state functional connectivity (RSFC) patterns obtained by DCBFC were compared with the anatomic axonal projection density (PDs) maps derived from the voxel-scale model. The results showed a high spatial correlation between RSFC patterns and PDs. ๑ The Authors. Published by SPIE under a Creative Commons Attribution 4.0 Unported License. Distribution or reproduction of this work in whole or in part requires full attribution of the original publication, including its DOI. [DOI: 10.1117/1 NPh.6.4.045014]
\end{abstract}

Keywords: fast clustering; functional connectivity; structural connectivity; resting state; calcium-sensitive optical imaging.

Paper 19049RR received Jun. 4, 2019; accepted for publication Nov. 20, 2019; published online Dec. 17, 2019.

\section{Introduction}

Spontaneous resting-state neural activity or hemodynamics has been used to reveal functional connectivity in the brain. ${ }^{1-5}$ The network topology of functional connectivity can deepen the understanding of the relationship between brain function and structure. Resting-state functional magnetic resonance imaging obtains whole brain cerebral blood oxygen level-dependent signals to analyze the functional connection and provides important references for diagnoses of neurophysiological disease. ${ }^{6-8}$ However, fMRI reflects hemodynamic fluctuations, which does not directly indicate neural activity. Investigating resting-state functional connectivity (RSFC) using direct neural signals, such as spontaneous fluctuation of calcium signals, can enable understanding of the mechanisms underlying the link between functional connectivity and brain structure. ${ }^{9-11}$ Because datasets for neural optical imaging usually have high spatiotemporal resolution, automatic fast clustering methods for widefield fluorescent calcium-signal imaging can help to improve the efficiency of RSFC analysis.

Current data-driven functional clustering methods can be grouped into two broad categories. The first is the nonclustering family, which includes algorithms based on probability distribution models, regional growth methods, decomposed signal and extracted components, self-organization mapping, dictionary learning, ${ }^{12}$ and edge detection. ${ }^{13}$ The probability density partition algorithm models the brain neural activity and uses an

*Address all correspondence to Pengcheng Li, E-mail: pengchengli@mail.hust .edu.cn optimization method to find the optimal solution. ${ }^{14-16}$ Regional growth chooses a few specific pixels or even all pixels in the cerebral cortex as seed points, and then the growth area is merged based on similar criteria until the region size is greater than the set threshold. ${ }^{17,18}$ The most common algorithm for extracting brain signal components used in fMRI is the independent component correlation algorithm, ${ }^{19-21}$ which extracts blind source information from original data and can perform denoising. This method requires repeated calculations to obtain the optimal number of components. Sparse decomposition generates as few atoms as possible to represent the original brain information by learning an overcomplete dictionary ${ }^{22,23}$ and can remove redundant information and improve analysis efficiency. Selforganizing maps show results as a neural network output layer. ${ }^{24}$

The other category is the clustering family that includes k-means, ${ }^{15,25-28}$ hierarchical clustering, ${ }^{28,29}$ spectral clustering, ${ }^{27,30-32}$ affinity propagation, ${ }^{23,33}$ fuzzy c-means, ${ }^{34}$ and density-based clustering. ${ }^{35,36}$ Some of these methods are sensitive to the initial selection (e.g., k-means, spectral clustering, and fuzzy c-means). As the same data may produce different clustering results, the method may repeat the calculation several times to obtain the best solution and require the iterative convergence to a cluster center. Some methods hierarchically integrate similar brain activity pixels into a cluster tree to identify the hierarchical relationship of clusters. However, it also leads to longer calculation time (e.g., hierarchical clustering). The methods based on density clustering treat clusters as high-density regions separated by low-density regions. ${ }^{35,36}$ Rodriguez and Laio $^{35}$ proposed a density-based algorithm that locates cluster centers using a criterion based on density peak, which can 
automatically calculate the cluster number and offers fast calculation. However, this method calculates the density for each pixel by summing the number of links whose geometric distances to the remaining pixels are less than the threshold, which is not suitable for analyzing the characteristics of neural activity signals, as the latter represents functional rather than distance clustering, and the correlation map of adjacent seed pixels are usually spatially continuous.

In this study, we propose a density center-based fast clustering algorithm (DCBFC) that is suitable for calcium-sensitive optical fluorescent imaging. To make the density peak-based clustering strategy useful for clustering the functional connectivity, the temporal Pearson's correlation is used to calculate the similarity between the time courses of pixel pairs in the DCBFC. Furthermore, a strategy of adaptive threshold is proposed for calculating the candidate cluster center based on the characteristics of in vivo calcium imaging data of a mouse cortex to improve the homogeneity of the intracluster and the heterogeneity of the intercluster. We tested our method using both simulated data and in vivo mouse data and compared our algorithm with the other clustering algorithms [k-means, hierarchical, spectral, principal components analysis (PCA)-k-means, PCA-hierarchical, and spectral-threshold clustering]. Using the adjusted Rand index (ARI) ${ }^{37}$ as a criterion, we evaluated the clustering performance of the algorithms on simulated data. The silhouette coefficient (SI) $)^{28,31,38-40}$ was used to quantify the homogeneity and heterogeneity of functional clusters on in vivo mouse data, and the processing time was also evaluated. The results show that the DCBFC has good performance and requires only a short calculation time. Finally, the functional connection maps obtained by the DCBFC are compared with the axonal projections labeled by recombinant adeno-associated virus (rAAV)-mediated tracing methods in Allen Mouse Connectivity. We used voxel-scale model ${ }^{41}$ to construct the whole isocortical projection connectivity at a scale of $100-\mu \mathrm{m}$ voxels. The average connection diversity/average connection strength (ACD/ACS) relationships of projection connectivity are calculated and compared with the ACD/ACS relationships of RSFC obtained by DCBFC. Because some clusters obtained by the DCBFC may cover more than one brain function area defined by cytoarchitecture, the main brain regions involved in each cluster are collectively referred to as "functional module" for convenience of expression. The codes used to generate the results are publicly available at https:/github.com/miuleee/ DCBFC.git and https://github.com/miuleee/FCvsPD.git.

\section{Materials and Methods}

\subsection{Density Center-Based Fast Clustering}

Our method attempts to rapidly obtain the functional parcellation by characterizing the cluster centers based on the following two criteria of density peak derived from Ref. 35 .

- A cluster center is assumed to be the cortical functional connection hub that tightly connects the surrounding pixels in a single functional module. The average Pearson's correlation coefficients between one central point, and its connected pixels should preferably be as high as possible.

- The correlation coefficient between two central hubs should be as low as possible. The temporal correlation coefficient between each pair of candidate centers must be smaller than the average correlation coefficient of any functional module.

Based on the two aforementioned properties, we propose an approach that attempts to directly obtain the central pixels by calculating a composite variable $\gamma$ Eq. (8), which represents the ability of a pixel to become a candidate center. A pixel with a greater $\gamma$ value is more likely to become a central point. A schematic of DCBFC is given in Fig. 1 and described as follows.

\subsubsection{Functional similarity matrix and threshold for determining cluster centers}

Neural signals in the same functional module fluctuate synchronously. After preprocessing of the measured data, we quantify the synchronization of calcium oscillations by calculating the functional similarity matrix [Fig. 1(c)]. Similarity between two pixels can be defined in many ways, and Pearson's correlation coefficient between time series of the recorded pixels was used to measure similarity in this study, given by the following equation:

$R_{t}(i, j)=\frac{\sum_{f=1}^{F}\left[p_{i}(f)-\overline{p_{i}}\right)\left(p_{j}(f)-\overline{p_{j}}\right]}{(F-1) \sigma_{i} \sigma_{j}}$,

where the functional similarity matrix $R_{t}$ is the temporal correlation coefficient matrix $(N \times N$, in which $N$ is the number of pixels), $R_{t}(i, j)$ is the correlation coefficient between pixels $i$ and $j, F$ is the frame number, $\bar{p}$ is the mean value of the time series for each pixel, and $\sigma$ is the standard deviation of $p$.

A threshold is applied to the similarity matrix to extract the strong connections used to determine the candidate central pixels. The threshold value for the correlation matrix will affect the final clustering results. An appropriate threshold should balance the compactness within a cluster and the heterogeneity between different clusters. To determine the threshold, clustering tests were conducted on the resting-state data of the calcium signal recorded from mice brains at 0.1 to 1 and 0.1 to $4 \mathrm{~Hz}$ filtering bands with thresholds ranging from $\left\langle\left|R_{t}\right|\right\rangle-\sigma\left(\left|R_{t}\right|\right)$ to $\left\langle\left|R_{t}\right|\right\rangle+$ $2 \sigma\left(\left|R_{t}\right|\right)$ at intervals of $0.025 \sigma\left(\left|R_{t}\right|\right)$, where $\left\langle\left|R_{t}\right|\right\rangle$ is the average of all elements in $R_{t}$ and $\sigma\left(\left|R_{t}\right|\right)$ is the corresponding standard

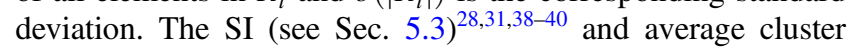
number are used as the measures for clustering performance. For the functional connectivity patterns obtained from the spontaneous calcium signal, a high SI value means that the correlation coefficients of the calcium-signal time series of the pixels contained in a functional module are as high as possible, and the correlation coefficients between the pixels in other functional modules are as low as possible. The closer the SI is to 1 , the better the clustering effect. As shown in Fig. 2, the blue and orange-dotted lines show average SI and average cluster number, respectively, for different thresholds, where the cluster number increases gradually with $\alpha$. We expected to be able to obtain a greater number of clustering numbers on the premise that its corresponding SI value is sufficiently large. The mean SI of all thresholds is shown as the green-dotted line in Fig. 2. It can be seen that the SI decreases at the beginning and approximately flattens around the green-dotted line in the interval from $\alpha=0$ to $\alpha=1$. It then gradually decreases as the threshold increases. We then chose the threshold value corresponding to the transition of the SI curve from the flat to the declining period [i.e., $\left.r t_{\text {thre }}=\left\langle\left|R_{t}\right|\right\rangle+\sigma\left(\left|R_{t}\right|\right)\right]$. The $r t_{\text {thre }}$ was used for both simulated 


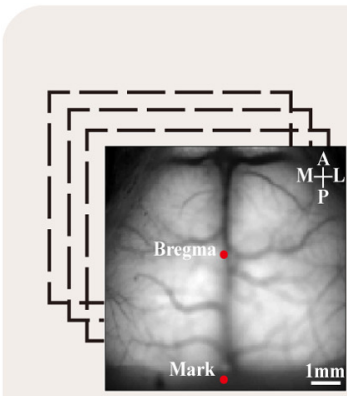

(a) Raw data

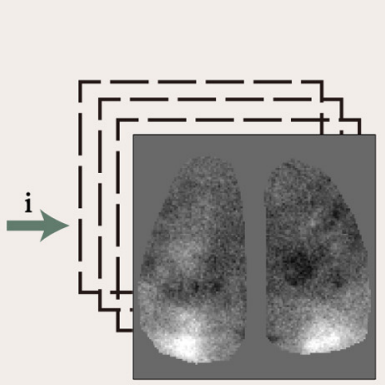

(b) Preprocess data

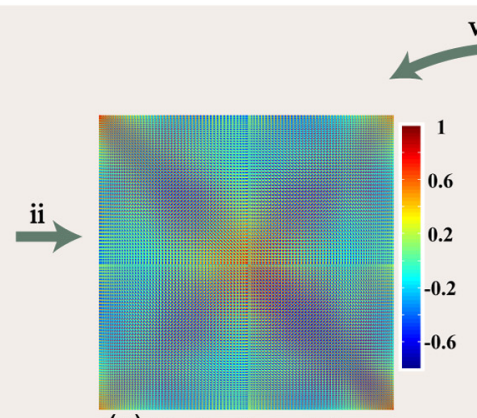

(c) Similarity matrix

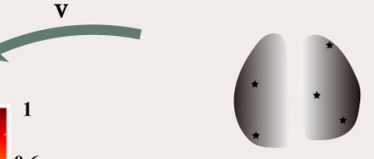

(e) Central pixels for current loop

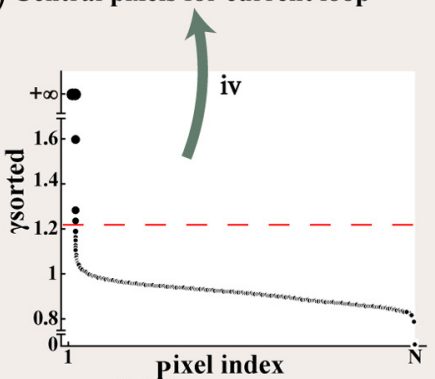

(d) Sort $\gamma$ value
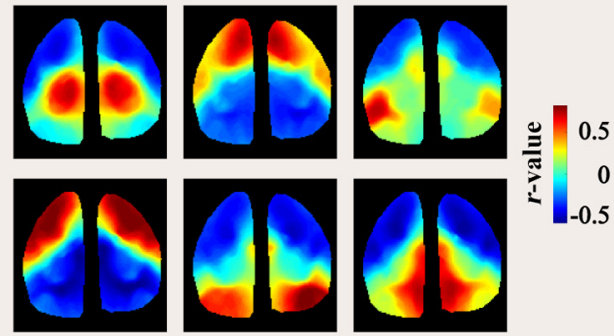

(h) Average functional connectivity patterns (r-value)

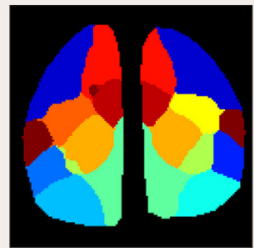

(g) Clustering all pixels

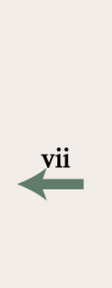

vii

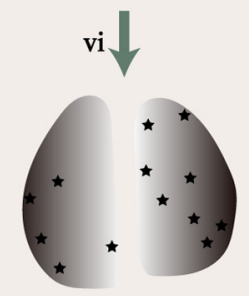

(f) All central pixels

Fig. 1 DCBFC Sketch. Step i: (a) Raw time-series fluorescent calcium images are acquired and processed with a $0.1-$ to $4-\mathrm{Hz}$ bandpass. Then, (b) the preprocessed images are obtained by global signal regression. Step ii: (c) The similarity matrix using Pearson's correlation between the time series is calculated. Then, a dataset $\{D\}=\{1, \ldots, N\}$ is initialized. Step iii: The composite index $\gamma$ values of all pixel are calculated, and (d) these values are sorted in descending order. The red-dotted line corresponds to the threshold $\gamma_{\text {thre }}$. The pixels whose $\gamma$ values are greater than $\gamma_{\text {thre }}$ are chosen as candidate central pixels. Step iv: The central pixels (e, black stars) of the current loop are screened out. Step v: Those pixels that are similar to the central pixels are removed from $\{D\}$, and the similarity matrix of the remaining pixels is used for the next loop. Steps iii-v: are repeated until no central pixels are available to select. Step vi: (f) All central pixels are obtained. Step vii: (g) All the other pixels in the image are assembled to the clusters corresponding to their central pixels. Step viii: The seed pixel functional connection maps corresponding to all pixels in the same cluster are averaged, and (h) the RSFC patterns of all clusters are obtained.

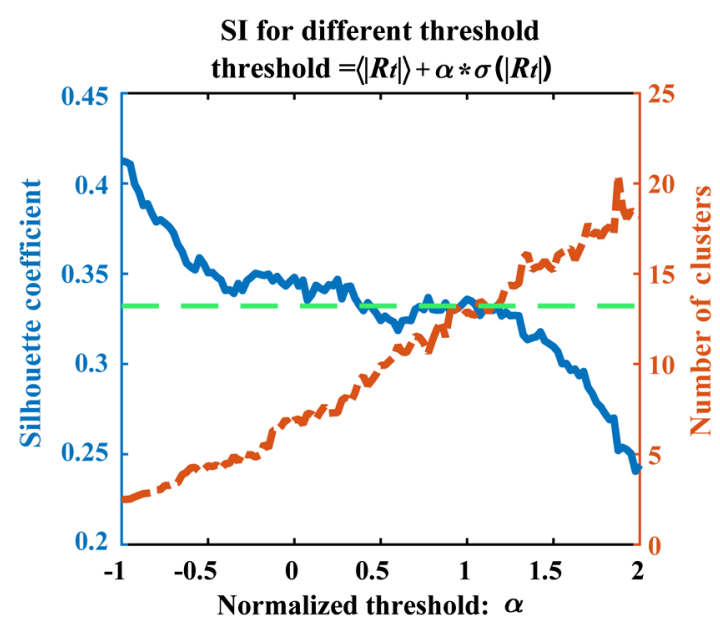

Fig. 2 Explore the threshold of DCBFC through experimental testing. Blue line and orange-dotted line, respectively, indicate average SI and average cluster number of different thresholds. Green-dotted line indicates the mean of all SI. In this study, the trading-off between $\mathrm{SI}$ and number of clusters was concerned. The threshold was set to be $\left\langle\left|R_{t}\right|\right\rangle+\sigma\left(\left|R_{t}\right|\right)$. and in vivo mouse data to ensure a high performance and large number of clusters simultaneously.

\subsubsection{Determining cluster centers}

Equations (2) and (3) were used to obtain the thresholded similarity matrix $R_{t}^{\prime}$, in which all the elements whose absolute values were below the threshold were set to 0 . The element in row $i$ and column $j$ of $R_{t}^{\prime}$ is denoted as $r_{i j}$, which represents the thresholded correlation coefficient between pixels $i$ and $j$ $(i, j \in[1, N])$. DCBFC clusters data by splitting the similarity matrix $R_{t}^{\prime}$. Here $H_{i}$ is the number of nonzero values of row $i$ 'th of $R_{t}^{\prime}$ given as in Eq. (4). If $H_{i}$ is lower than the cutoff index $n_{c}$ (usually set as $1 \%$ to $2 \%$ of $N^{35}$ ), then pixel $i$ may not be considered as a candidate cluster center. In Eq. (5), we set $\varphi\left(H_{i}\right)$ to zero to prevent pixel $i$ from being selected.

$$
\begin{aligned}
& r_{i j}=r_{i j} \chi\left(r_{i j}\right), \quad j \in[1, N], \\
& \chi\left(r_{i j}\right)= \begin{cases}1, & \text { if }\left|r_{i j}\right|>r t_{\text {thre }} \\
0, & \text { otherwise }\end{cases}
\end{aligned}
$$


$H_{i}=\sum_{j} \chi\left(r_{i j}\right)$

$\varphi\left(H_{i}\right)= \begin{cases}1, & \text { if } H_{i} \geq n_{c} \\ 0, & \text { otherwise }\end{cases}$

Two quantities were defined as criteria to determine the clustering center: the rank degree $\delta_{i}$ (the average of the absolute correlation coefficients that are greater than the $r t_{\text {thre }}$ between the $i$ 'th pixel and all other pixels) and the maximum correlation coefficient $\alpha_{i}$ between pixel $i$ and the other pixels whose $\delta$ values are higher than the $\delta$ value of pixel $i$. The reason we use the average correlation coefficient instead of the sum of multiple correlation coefficients is to ensure there is a chance for those pixels that have a specific seed-point correlation map, but with a small probability of occurrences in the correlation maps of all pixels, to be selected as cluster centers. Then, the pixel with the largest $\delta$ value is screened out and its $\alpha$ value is set to 0 .

$\delta_{i}=\frac{\varphi\left(H_{i}\right)}{H_{i}} \sum_{j}\left|r_{i j}\right|$,

$\alpha_{i}= \begin{cases}0, & \text { if } \delta_{i}=\max (\delta) \\ \left\{r_{i l} \mid l=\underset{j \in[1, N], \delta_{j}>\delta_{i}}{\arg \max }\left(r_{i j}\right)\right\}, & \text { otherwise. }\end{cases}$

Finally, a composite index $\gamma$ is defined as

$\gamma_{i}= \begin{cases}\infty, & \text { if } \Psi\left(\delta_{i}\right) \neq 0 \text { and } \Psi\left(\alpha_{i}\right)=0 \\ 0, & \text { or } \delta_{i}=\max (\delta) \\ \Psi\left(\delta_{i}\right) & \text { if } \Psi\left(\delta_{i}\right)=0 \text { and } \Psi\left(\alpha_{i}\right)=0 \\ \Psi\left(\alpha_{i}\right) & \text { otherwise }\end{cases}$

$\Psi(x)=\frac{x-\min (x)}{\max (x)-\min (x)}$,

where $\delta$ and $\alpha$ are normalized to the same orders of magnitude. The $\gamma$ of all pixels are sorted in descending order. The sorted $\gamma$ [Fig. 1(d)] rapidly falls off at the beginning and decreases slowly thereafter. This sorted $\gamma$ curve can be fitted by a formula such as $[k 1 \cdot \exp (k 2 / x)-\exp (-k 3 / x)] / k 4$ through an experimental test. Let $\gamma_{0}$ be the largest rational number of $\gamma$. We then calculate the threshold $\gamma_{\text {thre }}$ by the formula $\gamma_{\text {thre }}=\left(\gamma_{0}-1\right) / e+1$. Pixels with $\gamma$ values greater than $\gamma_{\text {thre }}$ are cluster central candidates. If $\Psi\left(\delta_{i}\right)$ and $\Psi\left(\alpha_{i}\right)$ both encounter $0, \gamma_{i}$ will be set to zero. This pixel may represent a slave point in one cluster.

To determine exhaustively the central pixels using the similarity matrix $R_{t}$, we initialize a dataset $\{D\}=\{1, \ldots, N\}$ that contains all pixels and perform a screening of the cluster centers in each loop: the pixels whose $\gamma$ are greater than $\gamma_{\text {thre }}$ are extracted from current dataset $\{D\}$ as candidate centers. Then, the correlation coefficients between all pairs of candidate centers are evaluated. For the two candidates whose correlation is greater than $r t_{\text {thre }}$, the candidate with a lower $\gamma$ will be removed from the list of candidate centers. The candidates that have not been deleted will then be selected as cluster centers. Before the next loop begins, the $n_{c}$ pixels that are most similar to each central point are removed from the dataset $\{D\}$ together with the determined cluster centers. In addition, the pixels whose correlation coefficient with the removed $n_{c}$ pixels is greater than $r t_{\text {thre }}$ are also removed from the dataset $\{D\}$. Then, the loop is repeated for new centers until all cluster centers are screened out (Fig. 1, steps iii to v).

\subsubsection{Clustering all pixels to form the functional modules}

After all cluster centers are obtained [Fig. 1(f)], all other pixels must be assembled to the clusters corresponding to the obtained central pixels. A pixel will be assigned to a center pixel with which it has the highest correlation among all the central pixels. Considering that the signal-to-noise ratio (SNR) of the spontaneous calcium activity signal with a single central pixel may be low, assembling a cluster based on it may reduce the compactness of the intracluster. Therefore, we used an averaged calcium signal for each cluster. To determine the pixel sets $\left\{C_{i}\right\}$ $(i=1,2, \ldots, k)$ used to obtain the averaged calcium signal, the $\gamma$ values of all the pixels of the image are first sorted in descending order. Then, the number of $m$ pixels (i.e., less than $1 \%$ of the total number of pixels) were allocated (i.e., according to the order of the $\gamma$ values, from high to low) to the central pixel if its correlation coefficient with the central pixel is greater than $r t_{\text {thre }}$. The mean spontaneous calcium activity signal over the $m$ pixels in the pixel set $\left\{C_{k}\right\}$ is regarded as the calcium activity signal sequence of the cluster corresponding to a central pixel. Then, each pixel is clustered into its most correlated cluster (i.e., with the highest correlation coefficient with the averaged calcium signal) [Fig. 1(g)]. Finally, the seed functional connection maps corresponding to all pixels in the same cluster are averaged to construct the RSFC of isocortex [Fig. 1(h)].

In this manner, DCBFC can automatically obtain the main resting-state functional modules, which makes further functional connection analysis more convenient.

\subsection{Data Preparation}

\subsubsection{Simulation data}

To evaluate the feasibility of DCBFC in processing resting-state optical imaging data, the simulated data were constructed using a linear superposition of the simulated cortical neural activity images and the background cortical images. The simulated cortical neural activity for each pixel consisted of multispike time series, as introduced by Pnevmatikakis et al. ${ }^{42}$ The background images were 1800 frames of autofluorescence images of restingstate C57BL/6J mouse cortex collected by the same optical imaging system used for the following calcium-signal imaging.

To validate DCBFC, we generated a simulated dataset containing the artificial functional modules as labeled ground truth in the following manner. First, we created a template pattern with a specific number of functional modules in the cerebral cortex and generated simulated cortical neural activity signals for each pixel. Pixels within the same module were highly correlated. Second, the background cortical images containing the physiological noises were superimposed on the simulated cortical neural activity images.

To evaluate the performance of DCBFC under different levels of SNR, we changed the amplitude ratio between the neural and background signals to obtain simulation data with different SNR ranges. Simulated data were also produced with different numbers of functional modules and different image sizes $(64 \times 64,128 \times 128$, and $256 \times 256)$ as follows.

i. Number of functional modules: 7 , SNR level: -16 to $10 \mathrm{~dB}$, time points: 1800 , repeat times: 3 . 
ii. Number of functional modules: 11, SNR level: $5 \mathrm{~dB}$, time points: 1800 , repeat times: 3 .

iii. Number of functional modules: 11, SNR level: $-10 \mathrm{~dB}$, time points: 1800 , repeat times: 3 .

iv. Number of functional modules: 50 , SNR level: $5 \mathrm{~dB}$, time points: 1800 , repeat times: 3 .

v. Number of functional modules: 50, SNR level: $-10 \mathrm{~dB}$, time points: 1800 , repeat times: 3 .

\subsubsection{Animal data acquisition and preprocessing}

All procedures were approved by the Committee for the Care and Use of Laboratory Animals at Huazhong University of Science and Technology. We generated Vglut2-GCaMP6s transgenic mice ( $n=4$, at approximately 6 weeks of age) by crossing the homozygous Vglut2-ires-cre (Jax. \#016963) and RCLGCaMP6s (Ai96; Jax. \#024106) strains. The presence of GCaMP expression was determined by genotyping each animal with polymerase chain reaction amplification.

Mice were anesthetized with isoflurane $\left(1.5 \%\right.$ in $\left.\mathrm{O}_{2}\right)$ and placed in a stereotaxic apparatus. The body temperature was maintained at $37^{\circ} \mathrm{C} \pm 0.5^{\circ} \mathrm{C}$. We made an intact skull window according to Ref. 43. A homemade fixed steel sheet was attached to the occipital bone with dental cement. The intact skull was directly covered with a thick layer of dental adhesive (C\&B-Metabond, Parkell, Edgewood, New York). Before the mixture became solid, a circular cover glass was gently placed on the skull without forming bubbles.

Widefield fluorescent calcium imaging was recorded through a transparent window $(\sim 8 \times 8 \mathrm{~mm})$. We focused the camera $\sim 400 \mu \mathrm{m}$ below the surface to reduce the effects of blood vessels. The cranial window was illuminated with a blue lightemitting diode (480 nm, FF01-480/40-25, Semrock), and the fluorescence GCaMP signal was acquired through a green light bandpass filter (530 nm, FF01-535/50-25, Semrock). We captured the spontaneous calcium-signal images using a SCMOS camera $(16$ bits, $6.5 \times 6.5 \mu \mathrm{m}$, HAMAMATSU ORCAFlash4.0 V3 C13440-20CU) at $10 \mathrm{~Hz}$ for 3 min each epoch.

A mouse cortical atlas was adapted from the Allen Institute Brain Atlas. We registered the Vglut2-GCaMP6s mice brain by marking bregma and an arbitrary marker on the sagittal suture according to the Allen Atlas. To remove the contribution of global physiological characteristics and other global fluctuations during imaging, the spontaneous activity of each pixel point in the recording sequence was subtracted from their mean fluorescence values for the entire time course. Then, a bandpass filter was used to the demean time courses to custom frequency bands temporally (e.g., 0.1 to $4 \mathrm{~Hz}$ ). Finally, we used global signal regression to remove potential global sources of noise (e.g., respiratory and cardiac noises). ${ }^{44}$

\subsection{Clustering Performance Evaluation}

The performance of DCBFC was assessed in terms of calculation time, receiver operating characteristic curve (ROC), ${ }^{45}$ ARI, ${ }^{37,39} \mathrm{SI}^{28,31,38-40}$ and Dice's coefficient. ${ }^{31,46}$ We used both simulation and animal experimental data to evaluate the performance of DCBFC and compared it with commonly used clustering methods, including k-means, hierarchical, and spectral clustering. It should be noted that DCBFC clustering uses a thresholded similarity matrix to calculate the candidate central pixels when determining the cluster center, which can be regarded as dimensionality reduction of data. For better evaluation of the speedup of DCBFC as compared with other algorithms, we used PCA to reduce the dimensionality of the time series for all pixel points for k-means and hierarchical clustering (referenced as PCA-k-means, PCA-hierarchical), in which the first $q$ principal components corresponding to $90 \%$ of the total eigenvalues were chosen, resulting in a reduction of the data from $N \times T$ to $N \times q$. For spectral clustering, singular value decomposition (SVD) was used to extract the eigenvectors of the Laplacian matrix and conduct k-means clustering for the extracted $K$ eigenvectors. Thus, SVD reduces the dimensionality from $N$ to $K$. In addition, we used the thresholded similarity matrix $R_{t}^{\prime}(N \times N)$ used in DCBFC as the weighted matrix for spectral clustering (referred to as spectral-threshold clustering).

All clustering programs described in the following sections were run on the same PC $\left[\right.$ Intel ${ }^{\circledR}$ Core $\left(\mathrm{Xeon}^{\circledR}\right)$ CPU E5-2687W v3@3.10 GHz, RAM $256 \mathrm{~GB}$ ], and all methods were tested using MATLAB code.

\subsubsection{ROC test}

The ROC curves (see Sec. 5.1) were plotted to analyze the relationship between artificial functional modules [Fig. 3(a)] and the functional connectivity maps obtained by DCBFC using the simulated data.

\subsubsection{ARI test}

Given the truth labels and clustering assignments, ARI (see Sec. 5.2) can measure the similarity between them. In the range of ARI as $[-1,1],-1$ indicates a bad clustering implement and 1 indicates a perfect match. Because the real clustering labels of simulation data are known, ARI is usually used to evaluate the performance of clustering algorithms with truth label assignments. K-means clustering, PCA-k-means clustering, and spectral clustering were repeated 50 times each turn, and the best clustering result was used. In this manner, we could compare the antinoise abilities and computation times of the different algorithms at diverse scales. We also tested the performance of seven clustering methods on different cluster numbers (see Fig. S1 in the Supplementary Material).

\subsubsection{SI and Dice's coefficient test}

Because no ground truth labels exist when clustering in vivo neural activity of brain regions, the clustering performance evaluation ARI is not applicable. Therefore, we adopted the SI (see Sec. 5.3) as a metric. $28,38-40$

To compare the reproducibility of the clustering results, Dice's coefficients for different methods were calculated to evaluate the similarity between the clustering results obtained at the group and individual levels. ${ }^{31,46}$ The range of Dice's coefficient was $[0,1]$, where 0 means no similarity and 1 means a complete match.

The functional modules were clustered to obtain the individual-level clustering result for each epoch. Each individual-level clustering result was then converted into an $N \times N$ adjacency matrix $A$, in which $A_{i j}=1$ if pixels $i$ and $j$ were in the same cluster; otherwise, $A_{i j}=0 .{ }^{31}$ All adjacency matrices of the individual level were subsequently summed to obtain group adjacency matrix $A g$. Then, the correlation coefficient matrix of $A g$ was clustered by DCBFC to obtain the clustering result for the group level. 


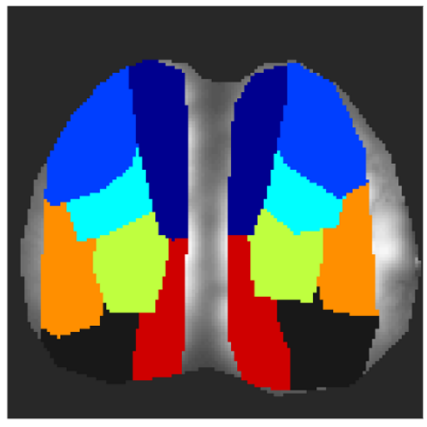

(a) Simulation template

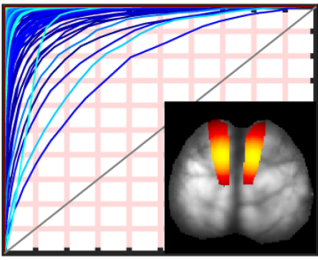

(b) Module 1

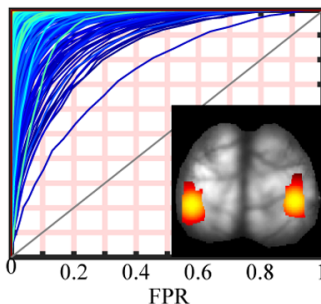

(f) Module 5

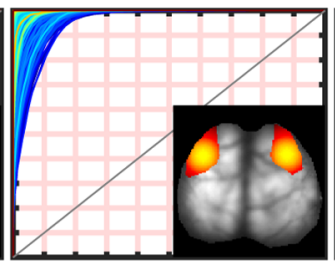

(c) Module 2

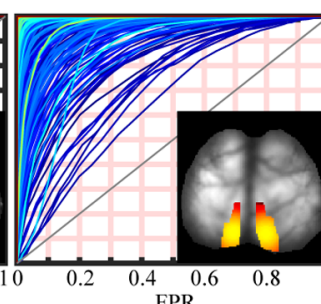

(g) Module 6

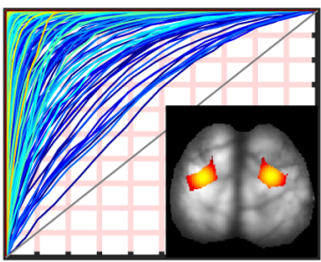

(d) Module 3

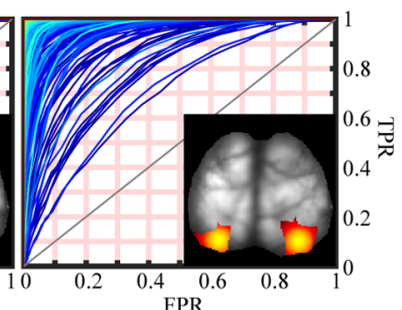

(h) Module 7

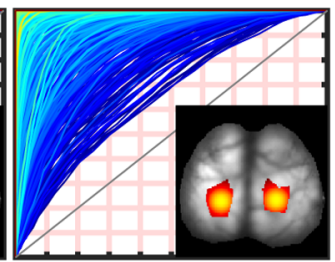

(e) Module 4

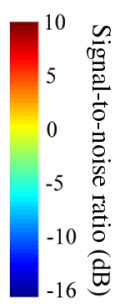

Fig. $3 \mathrm{ROC}$ analysis. Use ROC curves to analyze the relationships between artificial functional modules and functional connectivity maps with different SNR levels (SNR: -16 to $10 \mathrm{~dB}$ ). Averaged functional connection maps were generated for each $\mathrm{ROI}$ and seven series of ROC curves were plotted above. (a) Simulation template, (b) module 1, (c) module 2, (d) module 3, (e) module 4, (f) module 5, (g) module 6 , and (h) module 7.

\subsection{Comparing RSFC with Anatomic Connectivity of Axonal Projection}

To further validate the functional connectivity obtained, we compared RSFC with the anatomic connectivity of axonal projection obtained using the voxel-scale connectivity model proposed by Knox et al. ${ }^{41}$ to construct the whole isocortical connectivity at a scale of $100-\mu \mathrm{m}$ voxels. The model interpolates projection intensity in source space and enabled us to easily analyze the connectivity both in voxel and region scales.

The axonal projection data were downloaded from the Allen Mouse Brain Connectivity Atlas website ${ }^{47}$ the detailed information is listed in Table S2 in the Supplementary Material. Because no anatomy projection experimental data on the isocortex are found for the Vglut2-IRES-Cre line (widely expressed in most areas of the brain, except very sparse expression in the striatum and restricted populations within cerebellum, medulla, and pons), we used projection data from 185 experiments using several other types of mice, including C57BL/6J and excitatory Cre driver mice, which express in intratelencephalic neurons (project to both ipsilateral and contralateral cortex) ${ }^{48}$ All experiments used rAAV tracers for the labeling of axonal projections. These data were used to fit the voxel-scale model and derive the projection connectivity of the whole isocortex. Experiments were selected based on the following characteristics: (1) the experiment should not have large volume saturated in the injection region, and (2) the experiment should not have only few projection volumes outside of the injection site.

We used the Nadaraya-Watson connectome estimator with a radial basis function (RBF) kernel introduced by Knox et al. ${ }^{41}$ to estimate the projection connectivity (https://github.com/ AllenInstitute/mouse_connectivity_models). The voxel-scale connectivity model was constructed to include the connectivity projections from all voxels within the isocortical. The model fits the bandwidth of the RBF kernel by employing nested cross validation with held-out injection experiments.

To explore the relationship between RSFC and projection connectivity, we first calculated the spatial correlation between RSFC patterns obtained by different clustering methods and the projection density (PD) maps ${ }^{11}$ (see Sec. 5.5). We filtered the coordinate positions of mice isocortex according to information about brain regions provided in the Allen Mouse 3D Reference Model annotation and based on the coordinates of structure centers. The two-dimensional (2-D) normalized PDs were derived from the voxel-scale model ${ }^{41}$ and considered only the projection pathways of the brain regions on the isocortex. We compared the RSFC map with axonal PD patterns both in region and voxel scales: (1) the regional RSFC maps, $\mathrm{RSFC}_{\text {reg }}$, versus regional PD patterns, $\mathrm{PD}_{\mathrm{reg}}$ (Fig. 6). (2) The RSFC maps of central seed obtained by DCBFC, $\mathrm{RSFC}_{\mathrm{DCBFC}, \text { cen }}$, versus the $\mathrm{PD}$ patterns, $\mathrm{PD}_{\text {cen }}$, of the same cortical location (Fig. $\mathrm{S} 2$ in the Supplementary Material).

In addition, to explore quantitatively whether the functional links between the functional modules obtained by DCBFC had the anatomical connections, we calculated the functional connection strength (see Sec. 5.4) between different functional modules ${ }^{49,50}$ and compared them with the normalized projection connection density (see Sec. 5.6) between these functional modules. The average connection strength (ACS) of one functional module is used to quantify the overall strength of its connections across the whole isocortex. In addition, the average connection diversity (ACD) of different functional modules was calculated (see Secs. 5.4 and 5.6), which was derived from the Shannon entropy $^{51}$ and has been used in fMRI. ${ }^{49,50}$ Connection diversity quantifies the uncertainty that one functional module will connect to another. Module with low ACD prefers to connect with only a few functional modules. Module with high ACD trends to evenly connect with other regions across the whole isocortex. Here, "high" and "low" are relative values (i.e., relative to other functional modules, the connection strength or diversity is high or low). We normalized the ACS and the ACD to $[0,1]$ and regarded the normalized value that was greater than 0.5 as relatively high; otherwise, it was relatively low.

To conduct this analysis, DCBFC was used to obtain automatically the functional connection patterns of spontaneous neural activities in Vglut2- GCaMP6s mice (epoch $=6$ ), and the functional modules are formed at the group level. In the bilateral 


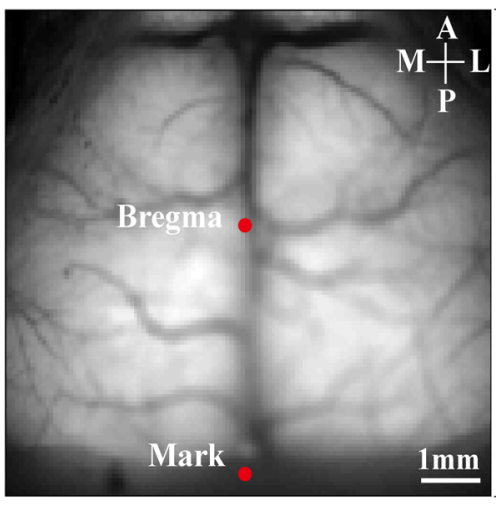

(a) Cortical window

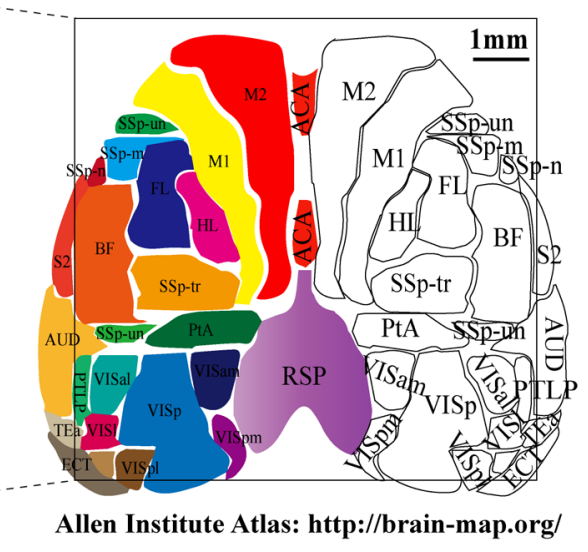

(b) Allen Mouse Brain Atlas
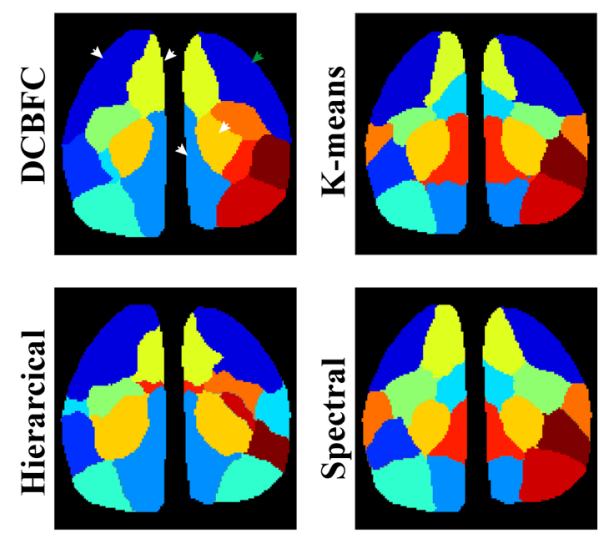

(c) Clustering results in group level

Fig. 4 Comparison with other existing methods for in vivo Vglut2-GCaMP6s mouse data. Two markers (bregma and one arbitrary marker) were made on the sagittal suture (a) and registered with the Allen atlas (b). (c) The clustering results of DCBFC, k-means, hierarchical, and spectral clustering at group level $($ epoch $=6)$. All pixels are color-coded based on the functional module to which they belong. Some functional modules may contain more than one brain region.

hemisphere, some symmetrical regions are clustered into one functional module [e.g., top of Fig. 4(c), white arrow], whereas others are divided into two. We merged the symmetrical regions as a functional module. Eight modules, represented as M2, M/S, BF, RSP/M, V, HL, FL, and PtA/SSp-tr, were then analyzed [Fig. 8(a)].

\section{Results}

\subsection{Clustering Performance Evaluation}

\subsubsection{ROC test}

Figure 3 provides the ROC curves of functional connectivity patterns obtained by DCBFC at different SNR levels in the simulated data. The results show that a strong correspondence existed between the functional connection map and simulated functional module, even when the SNR ratio is very low. For all modules [see Figs. 3(b)-3(h)], the clustering results and true labels had the strongest correspondence $(100 \%$ sensitivity and $100 \%$ specificity) in a broad range of thresholds ( 0.1130 to 0.7070 ) with SNR equal to $10 \mathrm{~dB}$. When the SNR was $-16 \mathrm{~dB}$, the strongest correspondence of module 1 [Fig. 3(b)] exhibited $73.8 \%$ sensitivity and $64.4 \%$ specificity. The values for the other regions (at $-16 \mathrm{~dB}$ ) were as follows: module 2 [Fig. 3(c), $91.6 \%$ sensitivity and $88.9 \%$ specificity], module 3 [Fig. 3(d), 68.2\% sensitivity and $50.5 \%$ specificity], module 4 [Fig. 3(e), $56.8 \%$ sensitivity and $62.4 \%$ specificity], module 5 [Fig. 3(f), $71.3 \%$ sensitivity and $65.8 \%$ specificity], module 6 [Fig. 3(g), 63.8\% sensitivity and $57.6 \%$ specificity], and module 7 [Fig. 3(h), 65.6\% sensitivity and 62\% specificity].

\subsubsection{ARI test}

Simulated cortical neural activity data with different image sizes $(64 \times 64,128 \times 128$, and $256 \times 256)$ were clustered using different methods. Table 1 shows the ARI values of the results obtained by the clustering algorithms for the aforementioned three image sizes, with $R_{t}$ as the similarity matrix. It was observed that DCBFC had significantly higher ARI values than those of k-means [two-way ANOVA tests, false discovery rate
(FDR) correction, $p<0.001$ ], spectral clustering $(p<0.001)$, PCA-k-means $(p<0.001)$, and spectral-threshold clustering ( $p<0.001$ for $64 \times 64, p<0.01$ for the others).

The clustering performances of different methods for various numbers of clusters $(4,11$, and 50 functional modules, respectively) are shown in Fig. S1 in the Supplementary Material. When the SNR level was greater than $-8 \mathrm{~dB}$, the ARI value of DCBFC approximated 1. With the addition of spatial background noise, DCBFC had the highest ARI value [blue line in Fig. S1(a) in the Supplementary Material].

\subsubsection{Functional parcellation obtained with in vivo data}

Figure 4 shows the group-level functional clustering results of the Vglut2-GCaMP6s mice data $(128 \times 128$ image size, epoch $=6$ ) using different clustering methods (DCBFC, kmeans, hierarchical, and spectral clustering). The results were registered in the Allen Mouse Brain Atlas [Fig. 4(b)] using the two markers on the skull [Fig. 4(a), red points]. In this study, DCBFC obtained 12 functional modules at the group level [upper left of Fig. 4(c), epoch =6]. Because the other clustering methods needed to set a specific number of clusters, the same number of functional modules as the DCBFC was used for k-means, hierarchical, and spectral clustering.

Figure 4(c) shows the functional modules clustered by DCBFC, k-means, hierarchical, and spectral clustering, and the corresponding functional connectivity maps are shown in the right of Figs. 6(a)-6(d). The functional connectivity maps illustrate that a correlation of spontaneous neural activity existed between the bilateral brain regions. Some regions that symmetrically connect between the bilateral hemispheres may have been clustered into the same functional connection module [upper left of Fig. 4(c), white arrow]. Some functional connection modules clustered by DCBFC were highly correlated to the anatomy brain areas (i.e., visual areas and primary somatosensory barrel field), whereas others may have covered more than one brain area, such as primary motor cortex, secondary motor cortex, and somatosensory area [upper left of Fig. 4(c), green arrow], which reflect the interconnection between different brain function areas. 
Table 1 Simulation testing: comparing the ARI of DCBFC with the other clustering methods using different picture size.

\begin{tabular}{lccccccc}
\hline Image size & DCBFC & K-means & Hierarchical & Spectral & PCA-k-means & PCA-hierarchical & Spectral-threshold \\
\hline $64 \times 64$ & $0.94 \pm 0.08$ & $0.76 \pm 0.10^{* *}$ & $0.95 \pm 0.07(p=0.989)$ & $0.81 \pm 0.03^{* *}$ & $0.74 \pm 0.08^{* *}$ & $0.90 \pm 0.10(p=0.050)$ & $0.89 \pm 0.10^{* *}$ \\
$128 \times 128$ & $0.99 \pm 0.02$ & $0.80 \pm 0.09^{* *}$ & $0.99 \pm 0.01(p=1.000)$ & $0.80 \pm 0.07^{* *}$ & $0.75 \pm 0.08^{* *}$ & $0.99 \pm 0.01(p=1.000)$ & $0.92 \pm 0.09^{*}$ \\
$256 \times 256$ & $0.99 \pm 0.01$ & $0.83 \pm 0.07^{* *}$ & $0.99 \pm 0.00(p=1.000)$ & $0.84 \pm 0.12^{* *}$ & $0.77 \pm 0.08^{* *}$ & $0.99 \pm 0.00(p=1.000)$ & $0.94 \pm 0.07^{*}$ \\
\hline
\end{tabular}

Note: Two-way ANOVA tests with FDR correction were used to compare the significant difference between ARI of DCBFC and the other methods. ${ }^{*} p<0.01$.

$p<0.001$.

\subsubsection{SI and Dice's coefficient test}

The average SI values of all experimental epochs for different clustering algorithms were $0.35,0.33,0.31,0.32,0.33,0.31$, and 0.34 , for DCBFC, k-means, hierarchical, spectral, PCAk-means, PCA-hierarchical, and spectral-threshold clustering, respectively, which indicate that the functional modules obtained by DCBFC had the highest synchronization between intercluster heterogeneity and intracluster homogeneity. The average Dice's coefficients under all experimental epochs for the aforementioned methods were $0.65,0.64,0.58,0.64$, $0.63,0.59$, and 0.64 , respectively, indicating that DCBFC had the highest reproducibility.

\subsubsection{Computation time}

Figure 5 illustrates the relative speedup performance of DCBFC compared to the other methods for three image sizes of the simulated data. We observed that hierarchical clustering, which links cluster objects step by step to generate a complete tree, had the longest computation time. The ratio of computing time between hierarchical clustering and DCBFC was in the range of 211.9 to $2855 \times$. Because k-means clustering must be calculated 50 times to obtain an optimal result, its calculation time was longer than that of DCBFC 3.7 to $16,542 \times$. Sometimes the iterations of k-means clustering were difficult to converge [Fig. 5(c), blue bar, 16,542×], which incurs a very long clustering time. The computation time for spectral clustering was affected by the number of functional modules, which was 1.1 to $133.2 \times$ longer than DCBFC in the case of 50-functional modules. After PCA was used to reduce the dimensionality of data, the computing times of k-means (PCA-k-means, Fig. 5, steel blue bar, 2.0 to $25.5 \times$ ) and hierarchical clustering (PCA-hierarchical, Fig. 5, sky blue bar, 41.7 to $2171 \times$ ) were greatly reduced. Spectral-threshold clustering (1.3 to $330.4 \times$ ) was not always faster than spectral clustering (Fig. 5 dark blue and black bars), which means that the threshold similarity matrix used in DCBFC was not the key to improving the speed. DCBFC still had a very short calculation time. In all cases, the calculation time of DCBFC was lower than those of the other methods.

Table 2 shows the average computing time of each algorithm for clustering Vglut2-GCaMP6s transgenic mice data. For the $128 \times 128$ image size, the computation speedup of DCBFC was $51.3,837.5,1.6,16.0,289.2$, and $1.5 \times$ faster than that of $\mathrm{k}$ means, hierarchical, spectral, PCA-k-means, PCA-hierarchical, and spectral-threshold clustering, respectively. To better demonstrate the advantages of DCBFC in terms of computing time, we clustered the images at a higher resolution $(256 \times 256$, epoch = 3). DCBFC was 13.0, 1406.0, 1.1, 2.1, 1308.1, and $1.6 \times$ faster than the other aforementioned methods, respectively.

\subsection{Relationship between RSFC and Axonal Projection Connectivity}

\subsubsection{Spatial correlation between isocortical axonal projection maps and RSFC maps}

In Fig. 6, the left plot of each subfigure shows the normalized regional $\mathrm{PD}$ maps $\mathrm{PD}_{\text {reg }}$ corresponding to 12 functional modules derived by the clustering methods [Fig. 4(c)]. The right side of the subfigures shows the RSFC maps corresponding to the modules. Figures 6(\#1)-6(\#12) represent M2 (secondary motor area), M/S (portions of motor areas combined with portions of primary somatosensory areas), L_BF (left primary somatosensory barrel field), R_BF (right primary somatosensory barrel field), HL (primary somatosensory lower limb), L_FL (left primary somatosensory upper limb), R_FL (right primary somatosensory upper limb), RSP/M (retrosplenial area combined with motor areas), L_V (left visual areas), R_V (right visual areas), L_PtA/SSp-tr (left posterior parietal association areas combined with primary somatosensory trunk), and R_SSp-tr/PtA (right posterior parietal association areas combined with primary somatosensory trunk). Figure 6(a) shows that all functional connectivity maps obtained by DCBFC had similar axonal projection patterns, in which the minimum and maximum spatial correlation coefficients were 0.75 and 0.91 , respectively. It is worthy to note that when the contralateral hemispheric connections existed in the RSFC map, the corresponding axonal projections also exhibited these long-range connections.

The spatial correlation coefficients between the regional RSFC maps obtained by different clustering methods and the corresponding regional PD patterns were calculated. The average spatial correlation coefficients for each method were 0.8288 (DCBFC), 0.8266 (k-means), 0.8156 (hierarchical), 0.8230 (spectral), 0.8271 (PCA-k-means), 0.8234 (PCA-Hierarchical), and 0.8262 (spectral-threshold). The average spatial correlation coefficient of DCBFC was slightly higher than that of other methods.

We also compared the RSFC maps of central pixels found using DCBFC with the PD patterns of the same cortical location obtained using the voxel-scale connectivity model, as shown in Fig. S2 in the Supplementary Material. The RSFC $\mathrm{DCBFC}_{\text {,cen }}$ and $\mathrm{PD}_{\text {cen }}$ patterns both showed a long-range link to contralateral or ipsilateral hemisphere for some brain regions. For example, we can see a functional connection between $\mathrm{M} 2$ and BF [Figs. S2(d)-S2(f) in the Supplementary Material] existing in both bilateral hemispheres, ${ }^{28}$ which can also be found in 


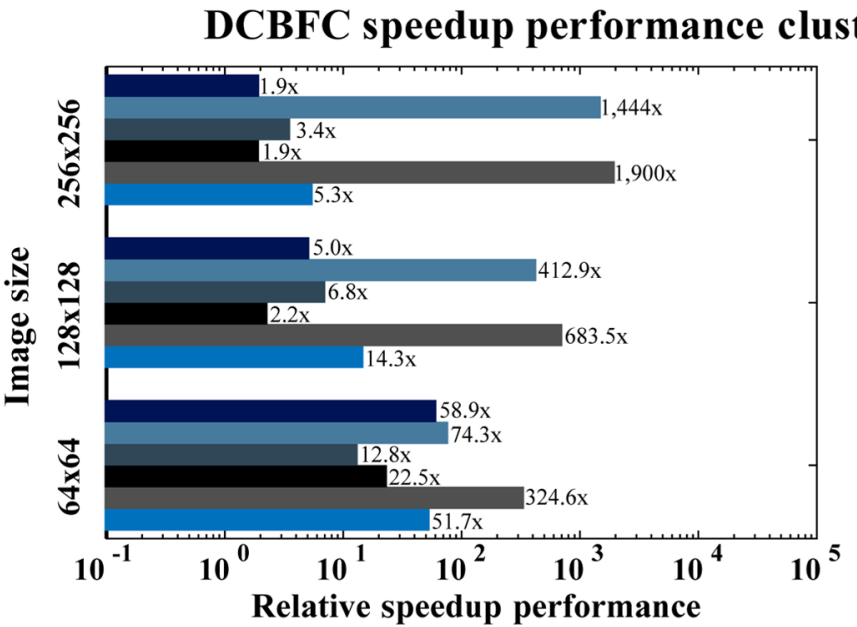

(a) 11-FunctionalModules \& SNR $=\mathbf{5} \mathbf{~ d B}$

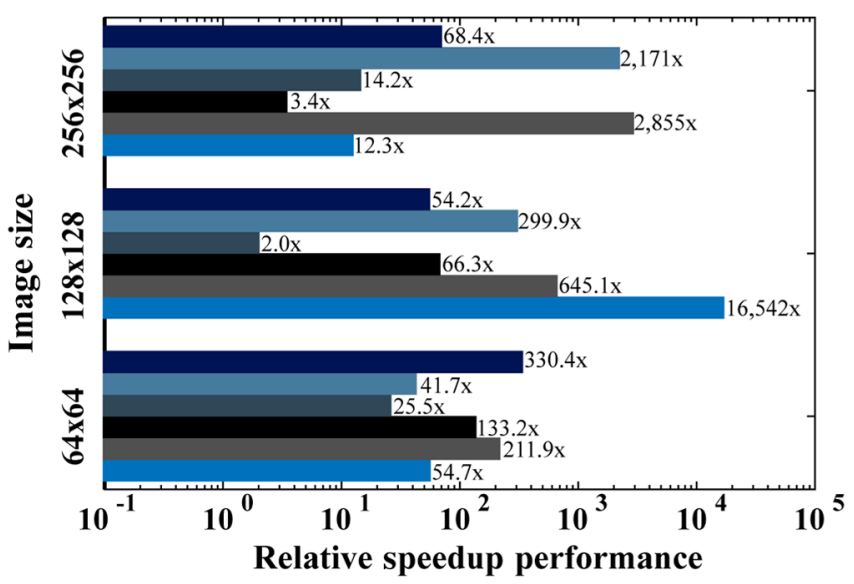

(c) 50-FunctionalModules \& $\mathrm{SNR}=\mathbf{5} \mathrm{dB}$

DCBFC vs k-means

DCBFC vs PCA-k-means
DCBFC vs hierarchical

DCBFC vs PCA-hierarchical

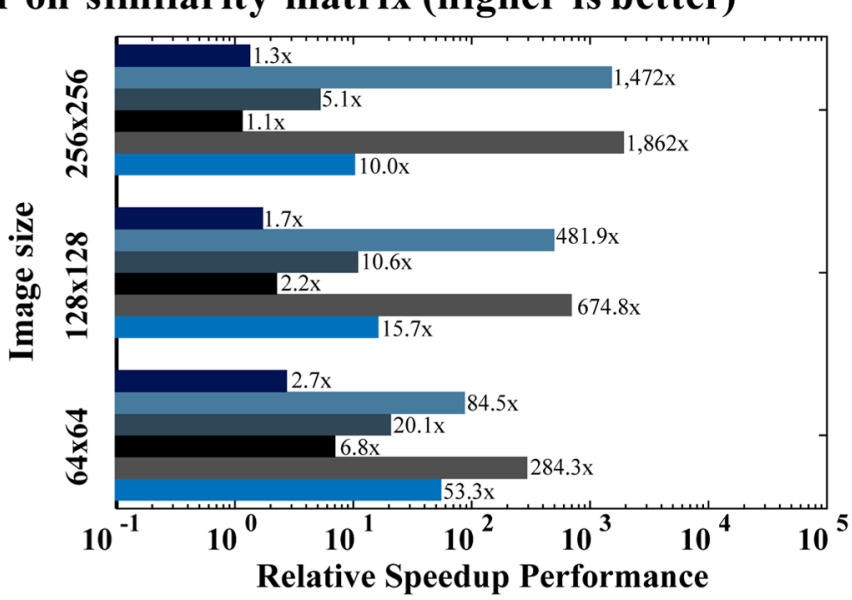

(b) 11-FunctionalModules \& SNR $=-10 \mathrm{~dB}$

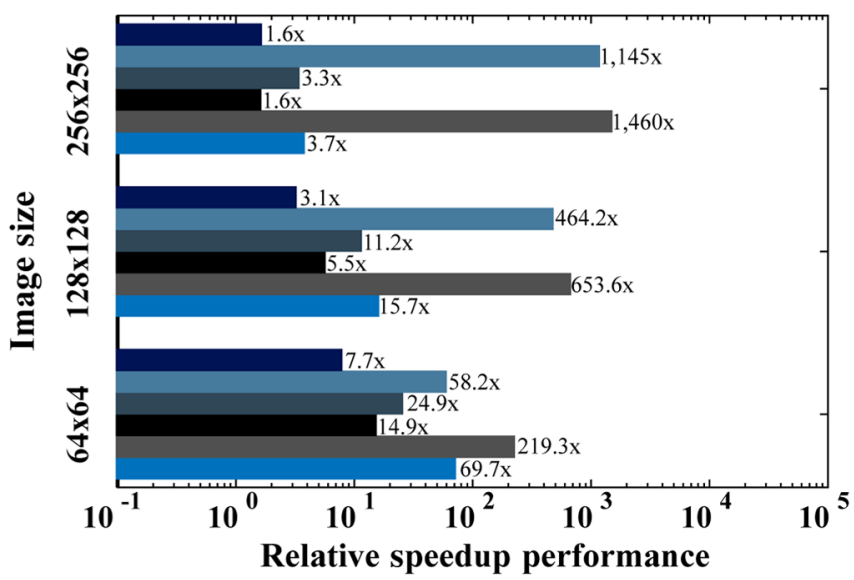

(d) 50-FunctionalModules \& SNR $=\mathbf{- 1 0} \mathrm{dB}$

DCBFC vs spectral

DCBFC vs spectral-threshold

Fig. 5 The relative speedup performance of DCBFC to the other methods (using $R_{t}$ as the similarity matrix). Data were tested on three types of image resolutions: $64 \times 64,128 \times 128$, and $256 \times 256$. (a) 11-functional modules type data with $5 \mathrm{~dB}$ SNR level. (b) 11 -functional modules type data with $-10 \mathrm{~dB}$ SNR level. (c) 50-functional modules type data with $5 \mathrm{~dB}$ SNR level. (d) 50-functional modules type data with $-10 \mathrm{~dB}$ SNR level. Compared with the other methods (k-means, hierarchical, spectral, PCA-k-means, PCA-hierarchical, and spectral-threshold clustering), DCBFC, respectively, provides a 3.7 to $16,542 \times$ performance boost over k-means clustering, a significant 211.9 to $2855 \times$ performance boost versus hierarchical clustering and almost 1.1 to $133.2 \times$ performance boost versus spectral clustering. Compared with k-means and hierarchical clustering using PCA, DCBFC also has 2.0 to $25.5 \times$ and 41.7 to $2171 \times$ performance boost. And has $1.3-330.4 \times$ performance gain versus spectral-threshold clustering.

Table 2 Experimental testing: comparing the average computing time of DCBFC to the other methods under different image size.

\begin{tabular}{cccccccc} 
Image Size & DCBFC & K-means & Hierarchical & Spectral & PCA-k-means & PCA-hierarchical & Spectral-threshold \\
\hline $128 \times 128$ & $5.6 \pm 0.4 \mathrm{~s}$ & $286.7 \pm 247.0 \mathrm{~s}$ & $4677.1 \pm 1565.9 \mathrm{~s}$ & $9.2 \pm 1.1 \mathrm{~s}$ & $89.3 \pm 92.6 \mathrm{~s}$ & $1614.9 \pm 66.0 \mathrm{~s}$ & $8.6 \pm 0.8 \mathrm{~s}$ \\
& & & & & & & \\
$256 \times 256$ & $79.8 \pm 1.6 \mathrm{~s}$ & $1038.0 \pm 166.7 \mathrm{~s}$ & $31.2 \pm 0.5 \mathrm{~h}$ & $88.3 \pm 2.0 \mathrm{~s}$ & $166.4 \pm 90.9 \mathrm{~s}$ & $29.0 \pm 1.2 \mathrm{~h}$ & $131.0 \pm 4.8 \mathrm{~s}$ \\
\hline
\end{tabular}

Note: Hierarchical and PCA-hierarchical were calculated in hours (h) at $256 \times 256$ image size. Others were measured in seconds (s).

the PD when the injection site of the PD map is M2 or BF. It should be noted that the average spatial correlation coefficient between the RSFC maps of the central pixels $\left(\mathrm{RSFC}_{\mathrm{DCBFC}, \mathrm{cen}}\right)$ obtained by DCBFC and the relevant normalized $\mathrm{PD}\left(\mathrm{PD}_{\mathrm{cen}}\right)$ patterns with the same location was lower than that between the regional RSFC maps $\left(\mathrm{RSFC}_{\mathrm{DCBFC}, \mathrm{reg}}\right)$ and regional normalized $\mathrm{PD}\left(\mathrm{PD}_{\text {reg }}\right)$ patterns $(0.69 \pm 0.07$ versus $0.83 \pm 0.05)$. In addition, the average spatial correlation coefficient between 


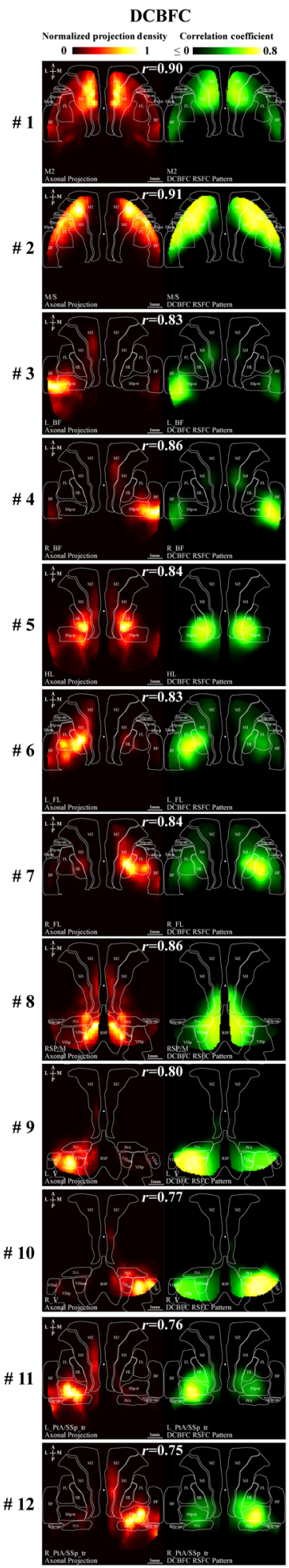

(a)

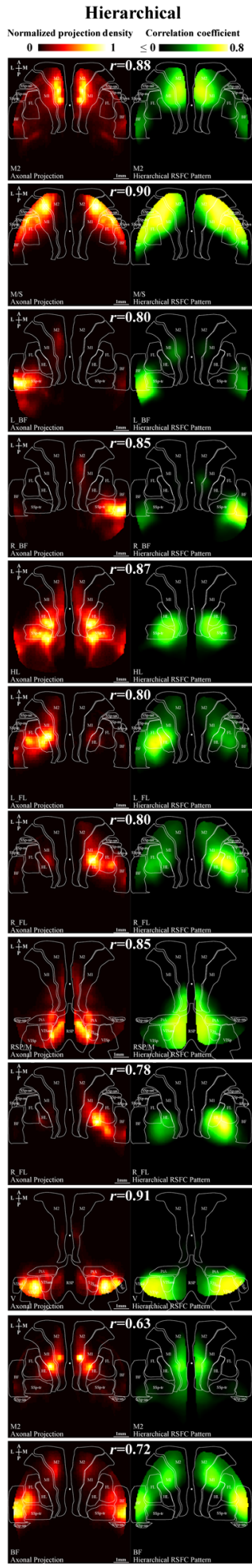

(b)

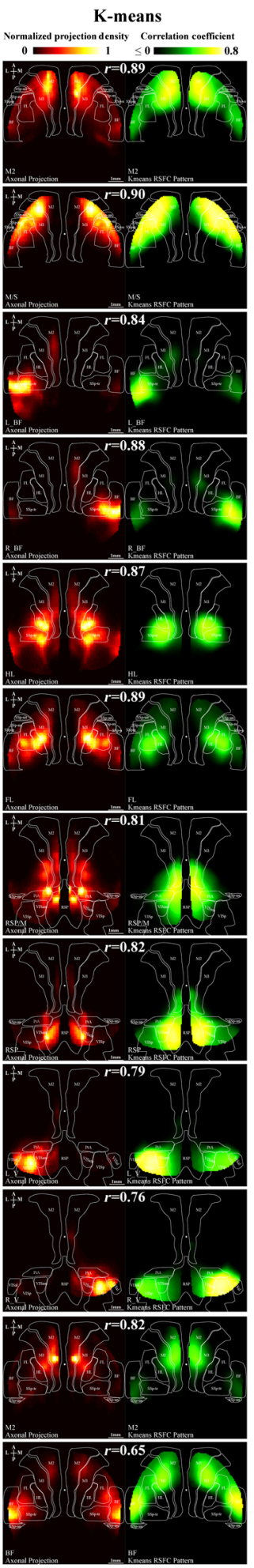

(c)

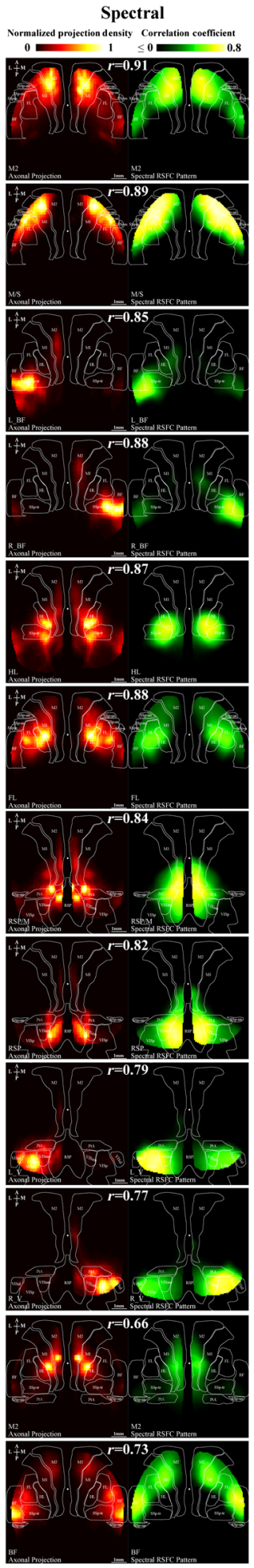

(d)

Structural connectivity versus functional connectivity

Fig. 6 Comparing the RSFC reg derived from different clustering methods (DCBFC, hierarchical, kmeans, and spectral clustering) with the $P D$ patterns (top views) $P D_{\text {reg }}$ derived from the voxel-scale model. (a)-(d) Left, the 2-D normalized isocortical PD maps constructed by the regionalized voxel-scale model, where the bottom left shows the injection_structure(s); right, functional connectivity maps obtained by clustering methods, where the lower left shows the brain region(s) covered by each functional connection module. The top of each pair of subfigures shows the spatial correlation coefficient of the two maps. Each row represents the connection maps for one of functional modules. The white circle represents the bregma mark. 
$\mathrm{RSFC}_{\mathrm{DCBFC} \text {,reg }}$ and $\mathrm{RSFC}_{\mathrm{DCBFC}, \mathrm{cen}}$ maps was $0.94 \pm 0.05$. Correspondingly, the average spatial correlation coefficient between $\mathrm{PD}_{\text {reg }}$ and $\mathrm{PD}_{\text {cen }}$ maps was $0.74 \pm 0.12$. The average spatial correlation between the RSFC maps and PD patterns across all seed pixels was $0.55 \pm 0.09$.

\subsubsection{ACS and ACD analysis of RSFC and axonal projection}

Figure 8(b) shows the resting-state connection strength $\operatorname{cs}(u, v)$ between the different functional modules clustered by DCBFC, where only significant connections were shown $(t$-test, FDR correction, $p<0.01$ ). Figure $8(\mathrm{c})$ shows the projection connection strength between different functional modules; only the connections whose PD is greater than $1.20 \times 10^{-04}$ are shown. The results reveal that all significant functional connections between functional modules clustered by DCBFC could be found in the axonal projection connectivity derived from the voxel scaling model. In addition, projection connectivity, shown in Fig. 8(c), had several additional connections (dashed line) that were not apparent in the resting-state functional connection, such as the connection between PtA/SSp-tr and M2, PtA/ SSp-tr and V, BF and HL, M2 and HL, and BF and V.

The functional connections between M2, M/S, FL, and BF shown in Fig. 8(b) were also found in previous studies. ${ }^{28,52,53}$ When M2 was the injection structure [Fig. 8(c)], the axonal projections were found to be transmitted to ipsilateral M/S, FL, BF modules, and the contralateral hemisphere [Fig. S2(d) in the Supplementary Material], which is consistent with the functional connection. M/S also had axonal projections toward FL. The injection site in motor area could project to SSp-m, FL, and the contralateral hemisphere [Fig. S2(a) in the Supplementary Material]. Table 3 shows that M/S closely connected only to certain isocortical functional modules in both RSFC and projection connectivity. The ACS between M/S (or M2) and all the other modules in the isocortex was relatively weak. The ACD of FL was relatively high both in RSFC and projection connectivity. ACS of FL was relatively high for RSFC but was relatively low for projection connectivity. BF could project to M2, PtA/SSp-tr, and FL and also had a long-range link to the contralateral hemisphere [Figs. 6(\#3) and 6(\#4)]. BF, HL, and PtA/SSp-tr had a relatively high ACS in terms of both RSFC and projection connectivity. PtA/SSp-tr was evenly connected to the other modules (high ACD). The projection pathway of the injection site in the visual cortex could be remotely connected to the posteromedial secondary motor areas [Figs. 6(a\#9) and 6(a\#10)], and this situation also occurred when the source and target sites were exchanged [Figs. 6(a\#8) and 8(c), RSP/M and V]. It is interesting that the RSP, which may correspond to the default mode network described in humans, ${ }^{28,49}$ showed both functional and projection connection with the visual, PtA/SSp-tr, HL, and posteromedial motor areas. Visual cortex preferentially connected to specific isocortical functional modules (e.g., link between V and RSP/M) in both RSFC and projection connectivity. ACD of RSP/M was relatively low for RSFC but was relatively high for projection connectivity. The ACS between RSP/M (or V) and all other modules in the isocortex was relatively weak. From Table 3, the results indicated that some modules with relatively high ACD (such as BF clustered by DCBFC) had few significant connections to other modules in Fig. 8.

\section{Discussion}

\subsection{Influence of Number of Clusters for DCBFC}

The other methods compared with DCBFC require a preset number of clusters. For the result described in Sec. 3.1.4, the clustering number of k-means was set as that obtained by DCBFC. To further discuss whether the clustering performance of DCBFC was also better than that of other methods for different number of clusters, the SI values of the results obtained by different methods were compared for the number of clusters ranging from 4 to 20. The number of clusters for DCBFC was changed by adjusting the threshold according to the method described in Table S1 in the Supplementary Material.

Figure 7 demonstrates SI values of brain functional modules clustered by DCBFC are higher than those clustered by k-means clustering $(p=0.0045)$, hierarchical clustering $(p<0.001)$, spectral clustering $(p<0.001)$, PCA-k-means clustering $(p=0.0150)$, PCA-hierarchical clustering $(p<0.001)$, and spectral-threshold clustering $(p<0.001)$ at a significance level of 0.05 (two-way ANOVA test with FDR correction).

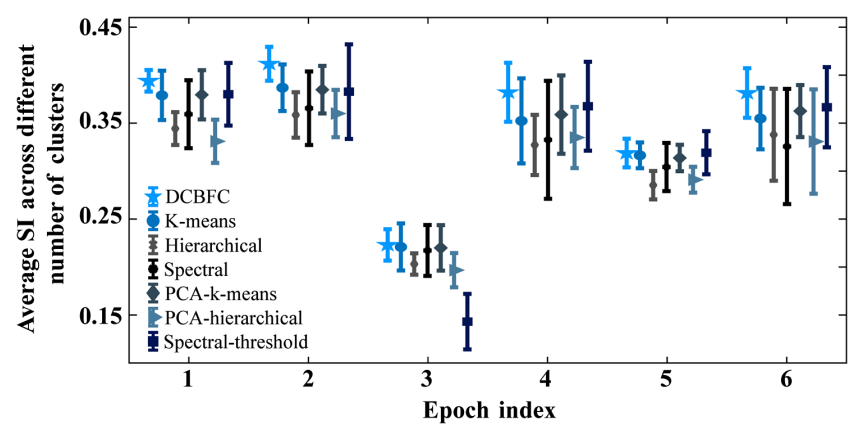

Fig. 7 DCBFC and other methods were compared for different number of clusters. The average SI values of different cluster numbers (from 4 to 20) of each method are shown $($ epoch $=6$ ).

Table 3 The normalized ACD and ACS of different modules.

\begin{tabular}{|c|c|c|c|c|c|c|c|c|}
\hline & $\mathrm{M} / \mathrm{S}$ & V & PtA/SSp-tr & M2 & $\mathrm{RSP} / \mathrm{M}$ & $\mathrm{BF}$ & $\mathrm{HL}$ & $\mathrm{FL}$ \\
\hline$A C D_{\text {norm, RSFC }}$ & 0.000 & 0.106 & 0.610 & 0.404 & 0.426 & 1.000 & 0.482 & 0.740 \\
\hline$A C D_{\text {norm,Proj }}$ & 0.270 & 0.000 & 0.847 & 1.000 & 0.588 & 0.474 & 0.868 & 0.723 \\
\hline ACS $_{\text {norm, RSFC }}$ & 0.450 & 0.000 & 1.000 & 0.488 & 0.495 & 0.504 & 0.950 & 0.895 \\
\hline ACS $_{\text {norm,Proj }}$ & 0.000 & 0.211 & 1.000 & 0.199 & 0.241 & 0.585 & 0.533 & 0.288 \\
\hline
\end{tabular}




\subsection{Influence of SNR on the Clustering Performance}

In this study, we tested the antinoise performance of different clustering methods and used $\mathrm{ARI}^{37}$ to evaluate the performances of different methods in clustering simulation data based on ground truth class assignment knowledge.

For simulation data, in the 4-functional modules test [see Fig. S1(a) in the Supplementary Material], with the addition of spatial background noise, DCBFC had the highest ARI values [blue line in Fig. S1(a) in the Supplementary Material] even at high noise levels. K-means clustering [green line in Fig. S1(a) in the Supplementary Material] and hierarchical clustering [red line in Fig. S1(a) in the Supplementary Material] clustered the data by calculating the correlation coefficients ( $r$ value) of each pair of time series, and they were influenced by the shape of the functional modules and background noise. This may have caused the pixels in different functional modules to cluster into the same module. Following PCA dimension reduction, the clustering performances of k-means [PCA-k-means, yellow line in Fig. S1(a) in the Supplementary Material] and hierarchical clustering [PCA-hierarchical, pink line in Fig. S1(a) in the Supplementary Material] improved, indicating that PCA dimension reduction was effective in eliminating spatial noise. For the other simulation data testing, without the addition of spatial background noise [see Table 1 and Figs. S1(b) and S1(c) in the Supplementary Material], spectral-threshold clustering, which employed the thresholded similarity matrix $R_{t}^{\prime}(N \times N)$ used in DCBFC as the weighted matrix for spectral clustering, had a higher ARI value than that of spectral clustering when using zero as the threshold for the similarity matrix (two-way ANOVA tests, FDR correction, $p<0.001$ for all).

\subsection{Time Consumption for Clustering}

DCBFC clustering used a thresholded similarity matrix to calculate the candidate central pixels during the step of determining the cluster center, which could be regarded as dimensionality reduction of data. To better evaluate the speedup of DCBFC compared with other algorithms, dimensionality reduction preprocessing was also applied to other clustering methods for comparison, such as PCA-k-means, PCA-hierarchical, and spectral-threshold clustering. The results showed DCBFC remained faster than other methods. Figure 5(c) shows that an abnormal calculation time existed for k-means (blue bar, 16,542×) which was much longer than other cases. This was because some clustering algorithms using iterative solution, such as k-means, spectral clustering, etc., may sometimes encounter slow iterative convergence due to the selection of initial value.

\subsection{Difference of Clustering Methods for Functional Parcellation}

Figure 4(c) reveals that the functional modules divided by different methods are slightly different. Compared to DCBFC, k-means and spectral clustering divided the RSP/M, M2, and $\mathrm{BF}$ regions into more subregions, hierarchical clustering did the same for the M2 and BF regions. DCBFC derived the PtA/SSPtr module, which was not differentiated from the other cortex regions by the other methods. The objective of DCBFC is to have the central pixels of different clusters be remote from each other and to aggregate all pixels based on their similarity to the central pixels. K-means and spectral clustering use the average time series of all the pixels in each cluster to update the current cluster center in each iteration so that they usually obtain higher intracluster correlation than DCBFC does. The average correlation coefficients between different functional modules derived using different methods were 0.40 (DCBFC), 0.46 (k-means), 0.43 (hierarchical), and 0.45 (spectral), which indicated the clusters obtained by DCBFC had largest distance between each other. The average similarities within the same functional module for different methods were 0.62 (DCBFC), 0.64 (k-means), 0.62 (hierarchical), and 0.63 (spectral). The intercluster similarity of DCBFC was the lowest, whereas k-means and spectral clustering were the highest (these relationships were still maintained for different number of clusters, data not shown). Therefore, k-means and spectral clustering obtain more subregions may be due to their high intracluster similarity and low intercluster distance.

Comparing the RSFC patterns of aforementioned subregions with the corresponding normalized PD maps [e.g., Figs. 6(a), 6(b)\#8 versus Figs. 6(c), 6(d)\#7, 6(d)\#8; Figs. 6(a)\#1 versus Figs. 6(b)-6(d)\#1, Figs. 6(b)-6(d)\#11], we can see that the average spatial correlation coefficients between $\mathrm{RSFC}_{\text {reg }}$ patterns and their corresponding $\mathrm{PD}_{\text {reg }}$ maps for these subregions were smaller than that for only one region.

As previously mentioned, from the perspective of functional clustering, when we hope that the functional connectivity module can be closely in contact within the same cluster and be far away from other clusters, SI can be used as an evaluation index for clustering performance. The results showed that DCBFC had the highest SI value and could rapidly cluster the connection matrix.

\subsection{Links between RSFC and Anatomy Projection Connection}

We describe the links between RSFC and anatomy projection connection from two respects.

1. The spatial correlation coefficients of two types of patterns reflect the spatial similarity between the two maps on the top view. The results [Fig. 6(a)] show that the spatial correlation coefficients between $\mathrm{RSFC}_{\mathrm{DCBFC} \text {,reg }}$ and corresponding $\mathrm{PD}_{\text {reg }}$ were in range of $[0.75,0.91]$. In addition, Fig. 8 shows that all the significant functional connections between functional modules can be found in the projection connectivity, whereas projection connectivity has several additional connections (dashed line) that are not shown in resting-state functional connection, such as the connections between PtA/SSp-tr and M2, PtA/SSp-tr and $\mathrm{V}, \mathrm{BF}$ and HL, M2 and HL, and BF and V. This may be partly due to the difference between the strains of mice used in projective connection fitting and that in RSFC calculating. It may also be because the axonal projection shows all existing anatomical output connection through axons, but RSFC here only shows the correlated spontaneous neural activity in the low frequency $(0.1$ to $4 \mathrm{~Hz})$. Moreover, the PDs connection between two adjacent brain regions such as the connection between $\mathrm{BF}$ and visual cortex might be overestimated when the virus is diffused from the injection site to its surrounding area if the dose of virus is not 


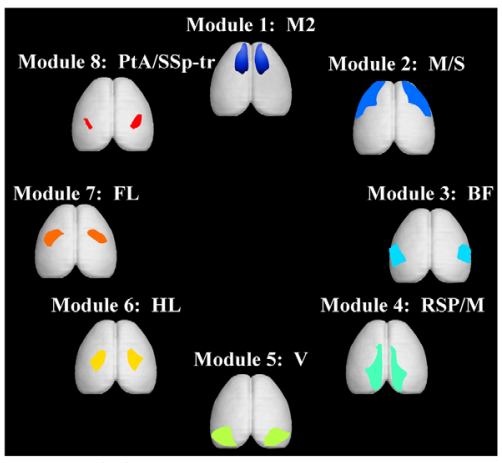

(a) Functional modules of the mice isocortex

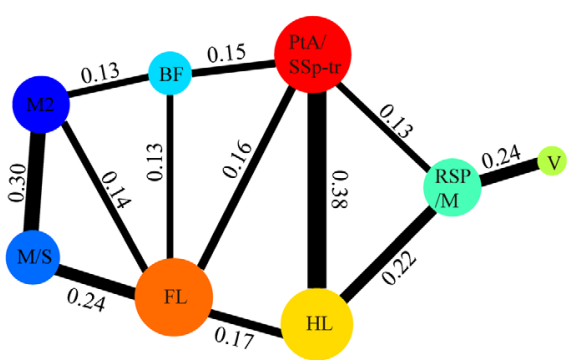

(b) Connection strength between different functional modules: RSFC

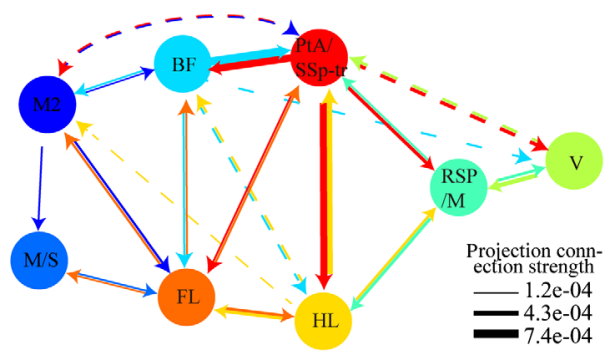

(c) Normlized connection density between different functional modules: projection connectivity

Fig. 8 Relationship between resting-state connectivity of Vglut2-GCamp6s mice and the axonal projection connectivity. (a) DCBFC was used to cluster mice data, and the two hemispherically symmetric functional modules were combined to form eight functional modules for analysis (M2, secondary motor cortex; M/S, motor areas and somatosensory areas; BF, barrel field cortex; RSP/M, retrosplenial area and motor cortex; V, visual area; HL, hindlimb region; FL, forelimb region; PtA/SSp-tr, parietal association areas and primary somatosensory trunk area). (b) The connection strength (undirected) between different isocortical functional modules. Note that this shows only significant connections ( $t$-test, FDR correction, $p<0.01$ ). The value on the link denotes the ACS between two modules. (c) A corresponding projection (directed) connectivity derived from the voxel-scale model shows only the connections with a normalized connection density greater than $1.20 \times 10^{-04}$. The color of the projection arrow is the same as the injection site. Dashed lines indicate the additional connections of the voxel-scale model that are different from DCBFC.

well controlled, or the location of virus injection may cover two adjacent function regions.

2. The ACD/ACS relationship is attempted to analyze whether the connection diversities of RSFC and projection connection are also at the same level (higher or lower) when their connection strengths are at the same level (stronger or weaker). It was shown that both ACD and ACS of axonal projection matched those of RSFC in some cortical regions, such as M/S, V, and PtA/SSp-tr. From Sec. 3.2.2, it could be seen that although some functional modules were closely connected to a few modules, their ACD values were relatively high, such as the BF for RSFC. This was partly because there were still weak connections between these modules and other modules were not shown besides those strong connections shown in Fig. 8 . Furthermore, ACD measures the difference of connection strengths between one functional module and all the other cortical modules based on Shannon entropy. If the difference of the connection strengths is large (ACD value is low), this module will trend to coactivity with certain specific modules. If the difference of connection strengths is small (ACD value is high), the connections of this functional module are spread evenly across the multiple brain regions.

\section{Appendix}

\section{$5.1 R O C$}

Given one artificial module, the values (true labels) of the pixels within this module were set to 1 and other pixels were set to 0 . Then the functional connection maps obtained by DCBFC were binarized with different thresholds (from -0.999 to 0.999 , with intervals of 0.001) at different SNR levels, in which the value of the pixel was set to 1 if its correlation coefficient was greater than the threshold value, whereas those of other pixels were set to 0 . Results were displayed using a series of ROC curves. For each SNR level, the corresponding true positive rate (TPR or sensitivity) versus false positive rate (FPR or 1-specificity) curve is plotted under the background of artificial functional modules (true label). In the equation $F P R=F P /(F P+\mathrm{TN}), F P$ denotes the number of pixels whose values are 1 in the binary functional connection map but whose true labels are 0 , and $T N$ denotes the number of pixels whose values are both 0 . In the equation $T P R=T P /(T P+F N), T P$ denotes the number of pixels whose values are both 1 , and $F N$ denotes the number of pixels whose values are 0 in binary functional connection map but whose true labels are 1 .

\section{$5.2 A R$}

Given the truth labels and the clustering assignments, ARI can measure the similarity between them. The range of ARI is $[-1,1]$, where -1 indicates a bad clustering implement and 1 indicates a perfect match.

$$
\mathrm{ARI}=\frac{\sum_{i, j}\left(\begin{array}{c}
n_{i j} \\
2
\end{array}\right)-\sum_{i}\left(\begin{array}{c}
n_{i \cdot} \\
2
\end{array}\right) \sum_{j}\left(\begin{array}{c}
n_{\cdot j} \\
2
\end{array}\right) /\left(\begin{array}{c}
n \\
2
\end{array}\right)}{\frac{1}{2}\left[\sum_{i}\left(\begin{array}{c}
n_{i \cdot} \\
2
\end{array}\right)+\sum_{j}\left(\begin{array}{c}
n_{\cdot j} \\
2
\end{array}\right)\right]-\sum_{i}\left(\begin{array}{c}
n_{i \cdot} \\
2
\end{array}\right) \sum_{j}\left(\begin{array}{c}
n_{\cdot j} \\
2
\end{array}\right) /\left(\begin{array}{c}
n \\
2
\end{array}\right)},
$$

where $n$ is the total number of pixels, $\left(\begin{array}{l}a \\ b\end{array}\right)=\frac{a !}{b !(a-b) !}$ represents the combinatorial number, $n_{i j}$ denotes the number of pixels at the intersection between artificial functional module $i$ and cluster $j, n_{i}$. denotes the number of pixels of artificial functional module $i$, and $n_{. j}$ denotes the number of pixels of cluster $j$. 


\section{$5.3 S I$}

The aim of SI is to identify whether the functional modules are compactly in contact with each other within the same cluster and are remote from interclusters. Suppose that $K$ is the ensemble of all clusters and pixel $i$ belongs to cluster $C_{i}$,

$$
\begin{aligned}
& \mathrm{SI}=\frac{1}{N} \sum_{i=1}^{N} \frac{b(i)-a(i)}{\max \{a(i), b(i)\}}, \\
& a(i)=\frac{1}{\left|C_{i}\right|-1} \sum_{j \in C_{i}}[1-r(i, j)], \\
& j \neq i \\
& b(i)=\min _{C_{l} \in K, i \notin C_{l}}\left\{\frac{1}{\left|C_{l}\right|} \sum_{j \in C_{l}}[1-r(i, j)]\right\},
\end{aligned}
$$

where $-1 \leq \mathrm{SI} \leq 1$, and SI increases when the heterogeneity between interclusters and the homogeneity within the same cluster increases simultaneously. Variable $a(i)$ is the average dissimilarity between pixel $i$ and all other pixels in its own cluster. Variable $b(i)$ is the minimum dissimilarity between pixel $i$ and all other interclusters, which means the average dissimilarity between pixel $i$ and the next nearest cluster for it.

\subsection{Connection Strength and Connection Diversity}

In this study, we only explored the positive correlation connections. The connection strength between modules $u$ and $v$, namely, $\operatorname{cs}(u, v)$, is defined as the average value of their positive time correlation coefficients given as

$\operatorname{cs}(u, v)=\frac{1}{N_{u} N_{v}} \sum_{i \in u} \sum_{j \in v} w_{i j}^{+}$,

where $N_{u}\left(\right.$ or $\left.N_{v}\right)$ is the number of pixels in module $u($ or $v)$ and $w_{i j}^{+}$denotes the positive time correlation coefficient between pixels $i$ and $j$. Let $K$ be the assemblage of all modules and $n$ the total number of modules. The ACS from module $u$ to the other regions is defined as $\mathrm{ACS}_{u}$, formulated as

$$
\operatorname{ACS}_{u}=\frac{1}{n-1} \sum_{v \in K, v \neq u} \operatorname{cs}(u, v) .
$$

The ACD for module $u$ is defined as $\mathrm{ACD}_{u}$, formulated as

$$
\mathrm{ACD}_{u}=-\frac{1}{\log (n-1)} \sum_{v \in K, v \neq u}\left(\frac{s_{u}(v)}{s_{u}} \log \frac{s_{u}(v)}{s_{u}}\right)
$$

$s_{u}(v)=\operatorname{cs}(u, v)$

$s_{u}=\sum_{v \in K, v \neq u} s_{u}(v)$,

where $\mathrm{ACD}_{u} \in[0,1]$. When module $u$ preferentially connects to a few other modules, the value of the connection diversity will be close to 0 . Module $u$ has a diversity value close to 1 when it trends to evenly connect with other modules across the cerebral cortex.

\subsection{Projection Density Map (Top Views)}

According to the code (i.e., the voxel-scale model) provided by Konx et al. (https://github.com/AllenInstitute/mouse_ connectivity_models), we derived PD maps for all functional modules ( $K$ PDs total) by summing and normalizing the PD value of all I to VI layers of isocortex. Therefore, the PDs were scaled to $[0,1]$. Finally, we derived $K$ PDs $\left(\mathrm{PD}_{\text {reg }}\right)$ corresponding to $K$ functional modules clustered by different clustering methods. In addition, we derived the $K$ cortical projection $\mathrm{PD}_{\text {cen }}$ maps whose injection site had the same cortical location with the $K$ central pixels found by DCBFC.

\subsection{Normalized Connection Density between Different Functional Modules}

The normalized connection density of projection connectivity was used in comparison with connection strength derived from RSFC. The projection connections from each voxel of the isocortex were first derived using the voxel-scale model. We then obtained the normalized connection density between different modules:

$\operatorname{cs}(u, v)=\frac{1}{N_{u} N_{v}} \sum_{i \in u} \sum_{j \in v} w_{i j}$

where $N_{u}\left(\right.$ or $\left.N_{v}\right)$ is the number of voxels located in module $u$ (or $v)$ and $w_{i j}$ indicates the PD value from voxel $i$ to voxel $j$. The average projection connection strength from module $u$ to the other modules $\left(\mathrm{ACS}_{u}\right)$ and the average projection connection diversity of module $u\left(\mathrm{ACD}_{u}\right)$ are given in Eqs. (15) and (16) in Sec. 5.4, respectively.

\section{Disclosures}

The authors have no relevant financial interests in this article and no potential conflicts of interest to disclose.

\section{Acknowledgments}

This work was funded by the National Natural Science Foundation of China (NSFC) (Nos. 61890951, 61775071, and 31471083); the National Key Research and Development Program of China (No. 2017YFB1002503); the Fundamental Research Funds for the Central Universities, Huazhong University of Science and Technology (No. 2019KFYXMBZ009); the Innovation Fund of Wuhan National Laboratory for Optoelectronics (WNLO). We thank the Allen Institute for Brain Science for providing a database of axonal projections.

\section{References}

1. M. P. Vanni and T. H. Murphy, "Mesoscale transcranial spontaneous activity mapping in GCaMP3 transgenic mice reveals extensive reciprocal connections between areas of somatomotor cortex," J. Neurosci. 34(48), 15931-15946 (2014).

2. A. W. Chan et al., "Mesoscale infraslow spontaneous membrane potential fluctuations recapitulate high-frequency activity cortical motifs," Nat. Commun. 6, 7738 (2015).

3. B. Li et al., "Coherent slow cortical potentials reveal a superior localization of resting-state functional connectivity using voltage-sensitive dye imaging," Neurolmage 91(8), 162-168 (2014).

4. B. Li et al., "Altered resting-state functional connectivity after cortical spreading depression in mice," NeuroImage 63(3), 1171-1177 (2012). 
5. Y. Ma et al., "Resting-state hemodynamics are spatiotemporally coupled to synchronized and symmetric neural activity in excitatory neurons," Proc. Natl. Acad. Sci. U.S.A. 113(52), E8463-E8471 (2016).

6. R. L. Buckner et al., "Cortical hubs revealed by intrinsic functional connectivity: mapping, assessment of stability, and relation to Alzheimer's disease," J. Neurosci. 29(6), 1860-1873 (2009).

7. C. Luo et al., "Altered functional connectivity in default mode network in absence epilepsy: a resting-state fMRI study," Hum. Brain Mapp. 32(3), 438-449 (2011).

8. K. Supekar et al., "Network analysis of intrinsic functional brain connectivity in Alzheimer's disease," PLoS Comput. Biol. 4(6), e1000100 (2008).

9. J. M. Stafford et al., "Large-scale topology and the default mode network in the mouse connectome," Proc. Natl. Acad. Sci. U.S.A. 111(52), 18745-18750 (2014).

10. J. Zimmermann, J. G. Griffiths, and A. R. McIntosh, "Unique mapping of structural and functional connectivity on cognition," J. Neurosci. 38(45), 9658-9667 (2018).

11. M. H. Mohajerani et al., "Spontaneous cortical activity alternates between motifs defined by regional axonal projections," Nat. Neurosci. 16(10), 1426-1435 (2013).

12. S. Zhao et al., "Supervised dictionary learning for inferring concurrent brain networks," IEEE Trans. Med. Imaging 34(10), 2036-2045 (2015).

13. A. L. Cohen et al., "Defining functional areas in individual human brains using resting functional connectivity MRI," NeuroImage 41(1), 45-57 (2008).

14. S. Ryali et al., "A parcellation scheme based on von Mises-Fisher distributions and Markov random fields for segmenting brain regions using resting-state fMRI," NeuroImage 65, 83-96 (2013).

15. Y. Golland et al., "Data-driven clustering reveals a fundamental subdivision of the human cortex into two global systems," Neuropsychologia 46(2), 540-553 (2008).

16. N. Honnorat et al., "GraSP: geodesic graph-based segmentation with shape priors for the functional parcellation of the cortex," NeuroImage 106, 207-221 (2015).

17. P. Bellec et al., "Identification of large-scale networks in the brain using fMRI," NeuroImage 29(4), 1231-1243 (2006).

18. Y. Lu, T. Jiang, and Y. Zang, "Region growing method for the analysis of functional MRI data," NeuroImage 20(1), 455-465 (2003).

19. J. A. Ash et al., "Functional connectivity with the retrosplenial cortex predicts cognitive aging in rats," Proc. Natl. Acad. Sci. U.S.A. 113(43), 12286-12291 (2016).

20. C. F. Beckmann and S. M. Smith, "Probabilistic independent component analysis for functional magnetic resonance imaging," IEEE Trans. Med. Imaging 23(2), 137-152 (2004).

21. L.-M. Hsu et al., "Constituents and functional implications of the rat default mode network," Proc. Natl. Acad. Sci. U.S.A. 113(31), E4541-E4547 (2016).

22. M. Zibulevsky and Y. Y. Zeevi, "Extraction of a source from multichannel data using sparse decomposition," Neurocomputing 49(1), 163-173 (2002).

23. T. Ren et al., "A novel approach for fMRI data analysis based on the combination of sparse approximation and affinity propagation clustering," J. Magn. Reson. Imaging 32(6), 736-746 (2014).

24. A. Mishra et al., "Functional connectivity-based parcellation of amygdala using self-organized mapping: a data driven approach," Hum. Brain Mapp. 35(4), 1247-1260 (2014).

25. S.-J. Hong et al., "The spectrum of structural and functional network alterations in malformations of cortical development," Brain 140(8), 2133-2143 (2017).

26. W. H. Jung et al., "Unravelling the intrinsic functional organization of the human striatum: a parcellation and connectivity study based on resting-state fMRI," PLoS One 9(9), e106768 (2014).

27. X. Shen, X. Papademetris, and R. T. Constable, "Graph-theory based parcellation of functional subunits in the brain from resting-state fMRI data," NeuroImage 50(3), 1027-1035 (2010).

28. M. P. Vanni et al., "Mesoscale mapping of mouse cortex reveals frequency-dependent cycling between distinct macroscale functional modules," J. Neurosci. 37(31), 7513-7533 (2017).

29. T. Blumensath et al., "Spatially constrained hierarchical parcellation of the brain with resting-state fMRI," NeuroImage 76, 313-324 (2013).
30. M. B. Nebel et al., "Disruption of functional organization within the primary motor cortex in children with autism," Hum. Brain Mapp. 35(2), 567-580 (2014).

31. R. C. Craddock et al., "A whole brain fMRI atlas generated via spatially constrained spectral clustering," Hum. Brain Mapp. 33(8), 1914-1928 (2012).

32. X. Zhang et al., "Individualized functional parcellation of the human amygdala using a semi-supervised clustering method: a 7T resting state fMRI study," Front. Neurosci. 12, 270 (2018).

33. J. Zhang et al., "Combining self-organizing mapping and supervised affinity propagation clustering approach to investigate functional brain networks involved in motor imagery and execution with fMRI measurements," Front. Hum. Neurosci. 9, 400 (2015).

34. A. Abrol et al., "Replicability of time-varying connectivity patterns in large resting state fMRI samples," NeuroImage 163, 160-176 (2017).

35. A. Rodriguez and A. Laio, "Clustering by fast search and find of density peaks," Science 344(6191), 1492-1496 (2014).

36. M. Ester, "A density-based algorithm for discovering clusters in large spatial databases with noise," in Proc. ACM Conf. Knowl. Discovery and Data Min., pp. 226-231 (1996).

37. L. Hubert and P. Arabie, "Comparing partitions," J. Classif. 2(1), 193218 (1985).

38. P. J. Rousseeuw, "Silhouettes: a graphical aid to the interpretation and validation of cluster analysis," J. Comput. Appl. Math. 20, 53-65 (1987).

39. F. Pedregosa et al., "Scikit-learn: machine learning in python," J. Mach. Learn. Res. 12, 2825-2830 (2011).

40. C. Kelly et al., "Broca's region: linking human brain functional connectivity data and nonhuman primate tracing anatomy studies," Eur. J. Neurosci. 32(3), 383-398 (2010).

41. J. E. Knox et al., "High-resolution data-driven model of the mouse connectome," Network Neurosci. 3(1), 217-236 (2019).

42. E. A. Pnevmatikakis et al., "Simultaneous denoising, deconvolution, and demixing of calcium imaging data," Neuron 89(2), 285-299 (2016).

43. G. Silasi et al., "Intact skull chronic windows for mesoscopic wide-field imaging in awake mice," J. Neurosci. Methods 267, 141-149 (2016).

44. K. Murphy et al., "The impact of global signal regression on resting state correlations: are anti-correlated networks introduced?" NeuroImage 44(3), 893-905 (2009).

45. J. A. Swets, Signal Detection Theory and ROC Analysis in Psychology and Diagnostics: Collected Papers, Lawrence Erlbaum Associates, Inc., Hillsdale, New Jersey (1996).

46. L. R. Dice, "Measures of the amount of ecologic association between species," Ecology 26(3), 297-302 (1945).

47. 2015 Allen Institute for Brain Science, Allen Mouse Brain Connectivity Atlas, http://connectivity.brain-map.org/.

48. J. A. Harris et al., "The organization of intracortical connections by layer and cell class in the mouse brain," bioRxiv, 292961 (2018).

49. A. Liska et al., "Functional connectivity hubs of the mouse brain," NeuroImage 115, 281-291 (2015).

50. M. Rubinov and O. Sporns, "Weight-conserving characterization of complex functional brain networks," NeuroImage 56(4), 2068-2079 (2011).

51. C. E. Shannon, "A mathematical theory of communication," Bell Syst. Tech. J. 27(3), 379-423 (1948).

52. B. R. White et al., "Imaging of functional connectivity in the mouse brain," PLoS One 6(1), e16322 (2011).

53. A. W. Bero et al., "Bidirectional relationship between functional connectivity and amyloid- $\beta$ deposition in mouse brain," J. Neurosci. 32(13), 4334-4340 (2012).

Miaowen Li is a PhD student in biomedical engineering at Wuhan National Laboratory for Optoelectronics, Huazhong University of Science and Technology, Wuhan, China. Her research focuses on optical imaging of cortical functional connectivity and machine learning.

Shen Gui is a PhD student in biomedical engineering at Wuhan National Laboratory for Optoelectronics, Huazhong University of Science and Technology, Wuhan, China.

Qin Huang Qin Huang received his PhD in optical engineering from Huazhong University of Science and Technology, Wuhan, China, in 2018 . 
Liang Shi is a $\mathrm{PhD}$ student in optical engineering at Wuhan National Laboratory for Optoelectronics, Huazhong University of Science and Technology, Wuhan, China.

Jinling Lu is an associate professor at Wuhan National Laboratory for Optoelectronics, Huazhong University of Science and Technology, Wuhan, China. Her research focuses on optical imaging of cortical functional connectivity.
Pengcheng $\mathbf{L i}$ is a professor of biomedical engineering, Huazhong University of Science and Technology. His research interests are in vivo functional optical imaging technologies of biological tissue, such as laser speckle imaging of blood flow and viscoelasticity, multispectral reflectance imaging of blood oxygenation, and multimodal optical imaging of brain functional activity. 